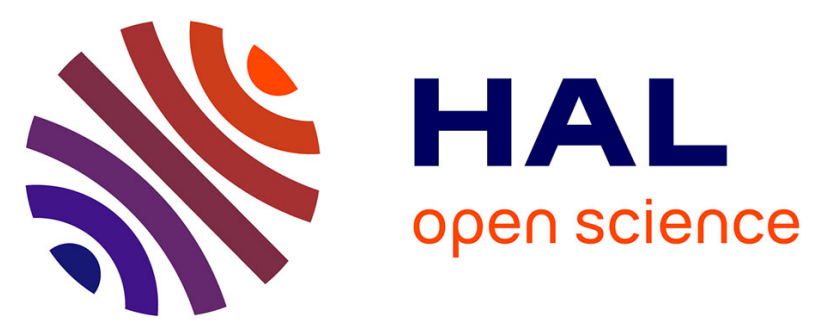

\title{
Assessment of diagnostic and prognostic condition indices for efficient and robust maintenance decision-making of systems subject to stress corrosion cracking
}

Khac Tuan Huynh, Antoine Grall, Christophe Bérenguer

\section{To cite this version:}

Khac Tuan Huynh, Antoine Grall, Christophe Bérenguer. Assessment of diagnostic and prognostic condition indices for efficient and robust maintenance decision-making of systems subject to stress corrosion cracking. Reliability Engineering and System Safety, 2017, 159, pp.237-254. 10.1016/j.ress.2016.11.022 . hal-01411368

\author{
HAL Id: hal-01411368 \\ https://hal.science/hal-01411368
}

Submitted on 8 Dec 2016

HAL is a multi-disciplinary open access archive for the deposit and dissemination of scientific research documents, whether they are published or not. The documents may come from teaching and research institutions in France or abroad, or from public or private research centers.
L'archive ouverte pluridisciplinaire HAL, est destinée au dépôt et à la diffusion de documents scientifiques de niveau recherche, publiés ou non, émanant des établissements d'enseignement et de recherche français ou étrangers, des laboratoires publics ou privés. 


\title{
Assessment of diagnostic and prognostic condition indices for efficient and robust maintenance decision-making of systems subject to stress corrosion cracking
}

\author{
K.T. Huynh ${ }^{\mathrm{a}, *}$, A. Grall ${ }^{\mathrm{a}}$, C. Bérenguer ${ }^{\mathrm{b}, \mathrm{c}}$ \\ ${ }^{a} I C D$, ROSAS, LM2S, Université de Technologie de Troyes, UMR 6281, CNRS, Troyes, France \\ ${ }^{b}$ Univ. Grenoble Alpes, GIPSA-lab, F-38000 Grenoble, France \\ ${ }^{c}$ CNRS, GIPSA-lab, F-38000 Grenoble, France
}

\begin{abstract}
Seeking condition indices characterizing the health state of a system is a key problem in condition-based maintenance. For this purpose, diagnostic and prognostic models have been unceasingly developed and improved over the past few decades; nevertheless none of them explains thoroughly the impacts of such indices on the effectiveness of maintenance operations. As a complement to these efforts, this paper analyzes the effectiveness of some well-known diagnostic and prognostic indices for maintenance decision-making. The study is based on a system subject to competing risks due to multiple crack paths. A periodic inspection scheme is used to monitor the system health state. Each inspection returns the perfect diagnostic information: the number of cracks, corresponding crack sizes, and the system failure/working state. Based on this information, two kinds of prognostic condition indices are predicted: the average value and probability law of the system residual useful life. The associated condition-based maintenance strategies and cost models are then developed and compared with the ones whose maintenance decisions are based on diagnostic condition indices. The comparison results allow us to conclude on the performance and on the robustness of these strategies, hence giving some suggestions on the choice of reliable condition indices for maintenance decision-making.
\end{abstract}

Keywords:

Condition-based maintenance, Diagnostic and prognostic condition index, Mathematical cost model, Residual useful life, Robust decision, Stress corrosion cracking

\section{Acronyms}

$\mathrm{CBM}$

$\mathrm{CM}$

SCC

HPP

HGP

DTS

RUL

MRL

PR

CR

pdf

cdf

\section{Notation}

$N_{t}$ condition-based maintenance

condition monitoring

stress corrosion cracking

homogeneous Poisson process

homogeneous Gamma process

degradation-threshold-shock

residual useful life

mean residual life

preventive replacement

corrective replacement

probability density function

cumulative distribution function

\footnotetext{
* Corresponding author

Email addresses: tuan.huynh@utt.fr (K.T. Huynh), antoine.grall@utt.fr (A. Grall), christophe.berenguer@grenoble-inp.fr (C. Bérenguer)
} 
$f_{T_{1}, \ldots, T_{k} \mid N_{t}}$

$1_{\{\cdot\}}$

$X_{\Delta t}^{i}$

$\alpha, \beta$

$m, \sigma^{2}$

$f_{\alpha \Delta t, \beta}, F_{\alpha \Delta t, \beta}$

$\Gamma(\cdot), \Gamma(\cdot, \cdot)$

$X_{t}^{S}$

$X_{t}^{S} \mid N_{t}$

$X_{[t, t+u]}^{S} \mid N_{t}, N_{t+u}-N_{t}$

$f_{X_{t}^{S} \mid N_{t}}, F_{X_{t}^{S} \mid N_{t}}$

$F_{X_{[t, t+u]}^{S}} \mid N_{t}, N_{t+u}-N_{t}$

$L, N$

$Z, Y$

$\rho\left(t \mid X_{t}^{S}, N_{t}\right)$

$R\left(t+u \mid X_{t}^{S}, N_{t}\right)$

$\mu\left(t \mid X_{t}^{S}, N_{t}\right)$

$T, \tau_{j}$

M

$N_{p}$

$\mu_{p}$

$R_{p}$

$\mathrm{PR}^{N}$

$\mathrm{PR}^{X}$

$\mathrm{CR}^{N}, \mathrm{CR}^{X}$

$Q_{\tau_{j}^{-}}, Q_{\tau_{j}^{+}}$

$C(t)$

$C_{\infty}$

$C_{i}, C_{p}, C_{c}$

$C_{d}$

$S_{1}$

$P_{p, k}(k T), P_{c, k}(k T)$

$W_{d, k}(k T)$

MTTF

$\eta\left(X_{t}^{S}, N_{t}\right)$

$\eta_{p}$

$M_{k}$

$\epsilon_{p}$

$\epsilon_{C}$ arrival time of the $i$-th crack

intensity of the HPP $\left\{N_{t}\right\}_{t \geq 0}$

joint pdf of $k$ first arrival times $\left\{T_{1}, \ldots T_{k}\right\}$ given $N_{t}$

indicator function

length of the $i$-th crack at $\Delta t$ units of time after its initiation

shape, scale parameters of the HGP $\left\{X_{t}^{i}\right\}_{t \geq 0}$

average rate, variance rate of $\left\{X_{t}^{i}\right\}_{t \geq 0}$

pdf, cdf of $X_{\Delta t}^{i}$

gamma function, incomplete gamma function

sum of all crack increments up to time $t$

sum of all crack increments in the time intervals $[0, t]$ given $N_{t}$

sum of all crack increments in the time intervals $[t, t+u]$ given $N_{t}$ and $N_{t+u}-N_{t}$

pdf, cdf of $X_{t}^{S} \mid N_{t}$

cdf of $X_{[t, t+u]}^{S} \mid N_{t}, N_{t+u}-N_{t}$

critical degradation threshold, limited number of cracks

failure times of the unmaintained system and of the maintained system

conditional RUL of the system at time $t$ given $X_{t}^{S}$ and $N_{t}$

conditional reliability of the system at time $t+u$ given $X_{t}^{S}$ and $N_{t}$

conditional MRL of the system at time $t+u$ given $X_{t}^{S}$ and $N_{t}$

inspection period, $j$-th inspection time

PR threshold associated with the sum of crack sizes

PR threshold of associated with the number of cracks

$\mathrm{PR}$ threshold of associated with the conditional MRL of the system

PR threshold of associated with the conditional reliability of the system

PR due to an excessive number of cracks over the threshold $N_{p}$

PR due to an excessive level of crack size over the threshold $M$

CR due to cracks number, CR due to sum of crack sizes

values of the quantity $Q$ at the time just before, and just after $\tau_{j}$

total maintenance cost including the downtime cost up to time $t$

long-run expected maintenance cost rate

cost per inspection, cost per PR, cost per CR

downtime cost rate

first replacement time of the system

probabilities of $\mathrm{PR}$ and $\mathrm{CR}$ at time $k T$

system downtime in a renewal cycle

mean time to failure of the system

either $\mu\left(t \mid X_{t}^{S}, N_{t}\right)$ or $R\left(t+T \mid X_{t}^{S}, N_{t}\right)$

either $\mu_{p}$ or $R_{p}$

minimum value of sums of crack sizes $x$ such that $\eta(x, k) \leq \eta_{p}$

relative error of $\mathrm{PR}$ decision parameters

relative increase in $C_{\infty}$ when the decision parameters are different than their optimal values

\section{Introduction}

Condition-based maintenance (CBM) program has nowadays attracted a great deal of interest among organizations, because of the significant economic impact to companies and environmental impact to society [1]. Within this maintenance program, condition monitoring (CM) information, such as vibration level, acoustic emission signal, temperature, pressure, humidity, etc., is collected and, through that knowledge, indices characterizing the health state of systems are synthesized 
for maintenance decision-making purposes. Such indices, called condition indices [2], may be the result of real-time diagnosis of impending failures or prognosis of future system health. In reality, diagnosis and prognosis are two essential aspects of a CBM program, and a lot of contributions have been developed in this field over the past few decades. Pusey and Roemer provide in [3] a broad overview of the development in diagnosis and prognosis applicable to high-performance turbo-machines until 1999. Jardine et al. give in [4] an interesting survey of the different diagnostic and prognostic approaches and associated CBM strategies for various machines up to 2005. Lee et al. [5] provide a comprehensive review of various methodologies and techniques in prognostics and health management research. More recently, Ahmad and Kamaruddin [6] and Shafiee et al. [7] systematically review maintenance decision-making methods based on the system health diagnostic and prognostic information. Other recent developments in this field can be found in [8-15]. A common remark drawn from these existing works is that different problems related to a CBM program, such as deterioration monitoring and health status assessment, remaining useful life (RUL) prediction, and maintenance decision-making, have been separately considered. This may lead to shortcomings in assessment of a whole CBM program. Furthermore, most efforts have been focused on improving the quality of diagnosis and prognosis processes; and the question on how to design and to use the best condition indices for maintenance decision-making remains an open and challenging issue. Faced to this situation, the present paper focuses on building a generic and complete framework to quantify the performance and robustness of CBM strategies built from some typical diagnostic and prognostic condition indices, and hence aims at providing suggestions on the choice of reliable indices for maintenance decision-making.

From an economic viewpoint, we define the performance of a maintenance strategy as its capacity to save maintenance costs under its optimal configuration, and its robustness as its ability to keep its cost saving close to the optimum when it is out of its optimal configuration. Classically, we can assess the maintenance strategy performance through its long-run expected maintenance cost rate [16]. Such a cost criterion can be analytically evaluated on the basis of (semi-)regenerative stochastic techniques $[17,18]$. In reality, the performance assessment based on the long-run expected maintenance cost rate is a key problem in the reliability and maintenance field, and it motivates many works on maintenance modeling and optimization in the literature [19]. Contrary to the problem of performance assessment, the robustness of maintenance strategies has not attracted much attention, especially for CBM strategies. As far as we know, only the authors of some recent papers [20-24] study the robustness of CBM strategies, and the assessment is mainly performed on the basis of the so-called relative increase in long-run expected maintenance cost rate [25]. By combining both above criteria, this paper proposes an approach to jointly quantify the performance and the robustness of CBM strategies, and thus to determine reliable condition indices for maintenance decision-making as follows

- developing a degradation and failure model for the considered system,

- synthesizing diagnostic and prognostic condition indices on the basis of the developed degradation and failure model,

- building diagnosis and prognosis-based maintenance strategies, and developing the associated cost models,

- assessing the performance and robustness of the considered strategies to find out reliable indices.

This approach allows exploring the complete CBM processing chain for a system, from deterioration monitoring and health status assessment, to remaining useful life estimation, right through to maintenance decision-making. It also constitutes a generic and complete framework to choose appropriate condition indices for CBM decision-making.

More precisely, we develop our degradation and failure model based on the operating feedback data-set described in [26]. This data-set consists of the initiation times and the crack length measurements in the propagation phase which appear on different components of nuclear power plants. The two-stage cracks behavior is thus modeled by two stochastic processes: a homogeneous Poisson process (HPP) for the incubation phase, and a homogeneous Gamma process (HGP) for the propagation phase. While such a use of these processes is quite classical [27], the way to define the competing failures of the system is new and original. Four condition indices are considered: sum of crack sizes, couple of sum of crack sizes and number of cracks, conditional mean residual life (MRL) of system, and system conditional reliability. The two first indices are directly gathered by inspection operations, while the two latter ones are synthesized using also the developed degradation and failure model. These indices represent respectively the partial and complete information about the current 
system heath, and the average type and quantile type information of the future system heath. These representative indices are then incorporated into maintenance decision-making. To maintain coherence with CM strategies recently implementing to obtain the operating feedback data-set [26], four quite simple periodic inspection and replacement strategies have been built. Note that the prognostic-based maintenance strategies proposed here are rarely considered in the literature. The mathematical cost models of these strategies are completely developed on the basis of the long-run expected maintenance cost rate, and of the classical renewal-reward theorem [28, page 39]. The quantitative comparisons on the performance and the robustness of these strategies allow us to find out reliable indices for maintenance decision-making.

As such, the main contributions and the originalities of this paper are fourfold.

- Exploring the complete CBM processing chain for a system, from condition monitoring to maintenance decision-making. Whereas different issues related to a CBM program are often investigated separately, we believe that we can get better insight into the CBM problem when considering them as a whole.

- Developing a new degradation and failure model for RUL prognosis of system subject to stress corrosion cracking (SCC) phenomena. This model is relatively generic because of its easy extension to other applications such as systems subject to the competing risks due to shock and degradation.

- Proposing new CBM strategies based on various variants of the system RUL (i.e., MRL and probability law of RUL), and developing their full mathematical cost models.

- Quantifying the effectiveness of diagnostic and prognostic condition indices for reliable maintenance decision-making purposes through the performance and robustness of the associated maintenance strategies.

The remainder of this paper is organized as follows. Section 2 is devoted to describing the operating feedback data-set based on which we develop the degradation and failure model of the system. Such a model allows to determine and analyze in Section 3 some diagnostic and prognostic condition indices for maintenance decision-making purpose. Section 4 deals with the detailed description and theoretical analyses of proposed CBM strategies. In Section 5, the associated maintenance cost models are developed and optimized. The assessment and discussions on the performance and robustness of the considered CBM strategies are carried out in Section 6. Finally, the paper is end with some conclusions.

\section{Description of Operating Feedback Data-Set, and System Modeling}

We aim at developing in this section a new degradation and failure model for systems subject to SCC. The modeling is inspired by the operating feedback data-set consisting of the initiation times and the measures of propagation length of cracks which appear on different components of nuclear power plants. For the best understanding of the model originality, we first present the operating feedback data-set and analyze associated models developed in the literature, then we describe in detail how to build our degradation and failure model. The original features and the applicability of the model are also analyzed at the end of the section.

\subsection{Operating Feedback Data-Set and Associated Models}

SCC which appears on different passive components or structures is a major source of degradation. Modeling this phenomenon has thus become a very important subject for the safety and reliability assessment issues. Laboratory tests indicate that SCC is characterized by three main stages [29]

- incubation - during this stage the crack has not yet appeared, and the stage ends when the environment becomes appropriate for SCC and the crack initiates,

- propagation - the crack appears and grows slowly until reaching a critical size,

- rupture - the crack grows quickly and leads to the failure of component. 
As argued in [26], the rupture stage is already critical, and any maintenance action made within this stage is useless because too late; therefore efforts have to focus on the modeling of crack behavior in the incubation stage and propagation stage. In reality, the modeling requires the information about the crack increments. So, the inspection date at which the crack is detected for the first time and the successive length measurements of a propagating crack after the detection time should be collected. As mentioned in [26], to build such a data-set for a nuclear power plant structure, ones have applied two particular CM strategies

- CM strategy 1 - $n$ passive components of the structure are periodically inspected, and only the information about the largest crack of each component is taken into account,

- CM strategy 2 - only one component of the structure is periodically inspected, and the information of all cracks appeared in the component is collected.

As a result, besides the initiation times of cracks, the data-set consists of the length measurements of the largest cracks (given by CM strategy 1) and of the "elementary" cracks (given by CM strategy 2). Such a kind of operating feedback data-set has inspired various degradation and failure models. Based on the data given by the first CM strategy, Grall et al. [30] assimilate a crack path to a HGP, while Huynh et al. [31] use a non-linear non-stationary Markov process obtained by the discretization of a Paris-Erdogan model. In their models, the incubation stage is ignored, and the length measurements of largest cracks are used to estimate the parameters of the Gamma process. In [26], Blain et al. model the crack evolution in both incubation stage and propagation stage by combining an exponential distribution and a Gamma process. The information on the cracks initiation times and on the largest crack length are jointly used for parameters estimation purpose. The data gathered by the second CM strategy motivate Castro et al. [27] to develop a model of multiple crack paths by coupling a HPP with various HGP, the failure occurs whenever the length of one crack path reaches a critical threshold. Then, the authors analyze an age-based maintenance strategy and a CBM strategy relied on such model in [32] and [33] respectively. Similar degradation and failure models can be found in [34-36]. One can remark from the above models that the data-sets given by the first and second CM strategies have been used separately. Since the data-set is very poor (only one crack increment and a few inspection times are gathered for one crack path [26]), they seem not enough reliable for safety and reliability analysis. It is then more interesting to develop a new degradation and failure model which can exploit the whole data-set given by both CM strategies. In the following, we attempt to build such a model.

\subsection{A Proposition for SSC Degradation and Failure Modeling}

Even though the degradation and failure model proposed here is inspired from the operating feedback data-set of a nuclear power plant [26], its applicability is not limited to nuclear power plant structures. This is why we use hereafter "system(s)" instead of "structure(s)" to highlight the generality of the model. As Singpurwalla advocated in [37], stochastic processes are very effective for modeling time-dependent degradation phenomenon like SCC, especially when the data-set is poor and uncertain. Sharing the same idea, we apply the two-stage stochastic process (i.e., HPP and HGP) to describe the behavior of a single-unit system subject to the competing risks due to multiple crack paths. The use of these processes in their homogeneous form for SCC modeling has been justified by many practical applications [19] and considered appropriate by experts [26]. Moreover, the homogeneous assumption allows us to facilitate the mathematical formulation and the estimation of model parameters in practice [35].

\subsubsection{Crack initiation modeling}

The system is assumed to start working at time 0 . The cracks appear in the system following a HPP with rate $\lambda, \lambda \in \mathbb{R}$ and $\lambda>0$. We denote $N_{t}, N_{t} \in \mathbb{N}$, the number of cracks up to time $t>0$, and $T_{i}, i=1,2, \ldots$, the arrival time (also called initial time) of the $i$-th crack where $T_{0}=0 . N_{t}$ is then a Poisson random variable with parameter $\lambda t$, and its probability mass function is given by

$$
P\left(N_{t}=k\right)=\mathrm{e}^{-\lambda t} \frac{(\lambda t)^{k}}{k !}
$$


The joint pdf of $k$ first arrival times $\left\{T_{1}, \ldots T_{k}\right\}$ given $N_{t}=k$ is obtained by [38, page 25]

$$
f_{T_{1}, \ldots, T_{k} \mid N_{t}}\left(t_{1}, \ldots, t_{k} \mid k\right)=\frac{k !}{t^{k}} \cdot 1_{\left\{0<t_{1}<\ldots<t_{k} \leq t\right\}}
$$

where $1_{\{\cdot\}}$ denotes the indicator function which equals 1 if the argument is true, and 0 otherwise.

\subsubsection{Crack propagation modeling}

Once a crack has been initiated, it propagates according to a HGP with shape parameter $\alpha>0$ and scale parameter $\beta>0$. Thus, let $X_{\Delta t}^{i}, i=1,2, \ldots$, be the length of the $i$-th crack at $\Delta t$ units of time after its initiation, the pdf and cdf of $X_{\Delta t}^{i}$ are given by [39]

$$
\begin{gathered}
f_{\alpha \Delta t, \beta}(x)=\frac{1}{\Gamma(\alpha \Delta t)} \beta^{\alpha \Delta t} x^{\alpha \Delta t-1} \mathrm{e}^{-\beta x} 1_{\{x \geq 0\}}, \\
F_{\alpha \Delta t, \beta}(x)=\frac{\Gamma(\alpha \Delta t, \beta x)}{\Gamma(\alpha \Delta t)},
\end{gathered}
$$

where $\Gamma(\alpha)=\int_{0}^{\infty} z^{\alpha-1} \mathrm{e}^{-z} d z$ and $\Gamma(\alpha, x)=\int_{0}^{x} z^{\alpha-1} \mathrm{e}^{-z} d z$ denotes the complete and incomplete Gamma functions respectively. The average propagation rate of each crack path is $m=\alpha / \beta$, and its variance rate is $\sigma^{2}=\alpha / \beta^{2}$.

Multiple crack paths appearing on the system may coalesce, accelerate the apparent crack growth, and hence generate largest cracks on the system [40]. We characterize such a phenomenon by two conditional random variables $X_{t}^{S} \mid N_{t}$ and $X_{[t, t+u]}^{S} \mid N_{t}, N_{t+u}-N_{t}$ :

- $X_{t}^{S} \mid N_{t}$ denotes the sum of all crack increments in the time intervals $[0, t]$ given the number of cracks up to time $t$, $N_{t}$. Its cdf and pdf are given by, see proof in Appendix A,

$$
\begin{gathered}
F_{X_{t}^{S} \mid N_{t}}(x \mid k)=P\left(X_{t}^{S}<x \mid N_{t}=k\right)=E_{\Theta}\left[F_{\alpha\left(k t-\sum_{i=1}^{k} \theta_{i}\right), \beta}(x)\right], \\
f_{X_{t}^{S} \mid N_{t}}(x \mid k)=\frac{d F_{X_{t}^{S} \mid N_{t}}(x \mid k)}{d x}=\frac{d E_{\Theta}\left[F_{\alpha\left(k t-\sum_{i=1}^{k} T_{i}\right), \beta}(x)\right]}{d x}=E_{\Theta}\left[f_{\alpha\left(k t-\sum_{i=1}^{k} \theta_{i}\right), \beta}(x)\right],
\end{gathered}
$$

where $f_{\alpha\left(k t-\sum_{i=1}^{k} \theta_{i}\right), \beta}(x)$ and $F_{\alpha \cdot\left(k t-\sum_{i=1}^{k} t_{i}\right), \beta}(x)$ are derived from (3) and (4) respectively, $E_{\Theta}[\cdot]$ denotes the expectation with respect to the random vector $\Theta=\left\{\theta_{1}, \ldots, \theta_{k}\right\}$ whose each element follows a uniform distribution $\theta_{j} \sim U(0, t)$, $j=1, \ldots, k$.

- $X_{[t, t+u]}^{S} \mid N_{t}, N_{t+u}-N_{t}$ represents the sum of crack increments in the time intervals $[t, t+u]$ given the number of cracks appeared at time $t, N_{t}$, and given the additional number of cracks in the time interval $[t, t+u], N_{t+u}-N_{t}$. The cdf of this variable can be computed as, see proof in Appendix B,

$$
F_{X_{[t, t+u]}^{S} \mid N_{t}, N_{t+u}-N_{t}}(x \mid k, h)=P\left(X_{[t, t+u]}^{S}<x \mid N_{t}=k, N_{t+u}-N_{t}=h\right)=E_{\Theta}\left[F_{\alpha\left((k+h) u-\sum_{i=1}^{h} \theta_{i}\right), \beta}(x)\right]
$$

where $F_{\alpha\left((k+h) u-\sum_{i=1}^{h} \theta_{i}\right), \beta}(x)$ is derived from (4).

\subsubsection{Failure modeling}

Many tests and practical applications show that the system fracture is mostly caused by coalescence of multiple cracks rather than by growth of some primary cracks [41]. This is why we consider that the system fails whenever the sum of crack increments up to time $t, X_{t}^{S}$, exceeds a critical degradation threshold $L$ or the number of cracks in the time intervals [0, $t$, $N_{t}$, reaches a limited number of cracks $N$. So, let $Z$ be the system failure time, it can be expressed as

$$
Z=\inf \left(t \geq 0, X_{t}^{S} \geq L \text { or } N_{t} \geq N\right)
$$

To illustrate the degradation and failure behavior of the considered system, we use the following set of parameters: $\alpha=1$, $\beta=1, \lambda=0.5, L=25, N=6$. In Fig. 1a, the sum of all crack sizes exceeds the threshold $L=25$ before reaching the limited number of cracks $N=6$, the system failure is then due to an excessive level of crack size. In Fig. 2b, we observe an opposite phenomenon and the system fails because of an excessive number of cracks. 


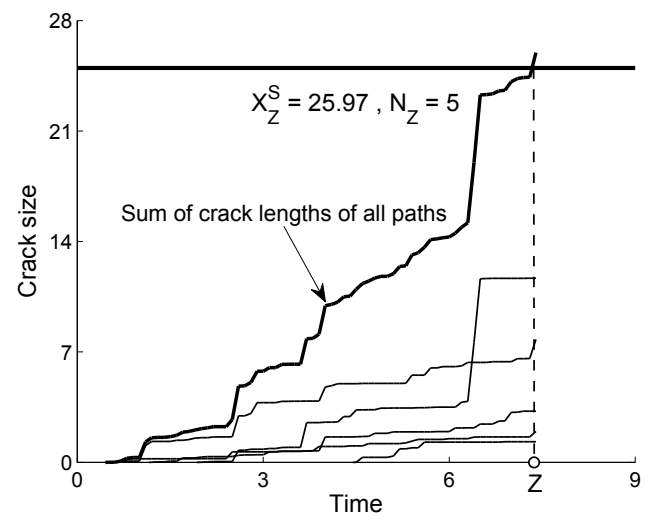

(a) Failure due to an excessive level of $X_{t}^{S}$.

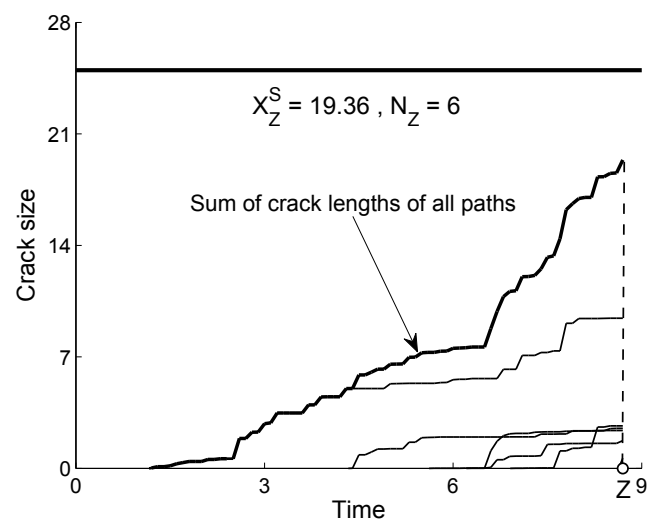

(b) Failure due to an excessive number of $N_{t}$.

Fig. 1 - Illustration of the competing failures in the system - $\alpha=1, \beta=1, \lambda=0.5, L=25, N=6$.

\subsection{Model Original Features and Applicability}

We have developed a degradation and failure model based on the statistical and physical properties of SCC. Unlike classical works, our model characterizes the behavior of both primary and largest cracks; this allows us to use the whole data given by both the considered CM strategies to estimate and verify the model parameters. As such, our model exploits the operating feedback data-set better than the aforementioned classical models. This promises then a reliable model for safety and reliability analysis. The model parameters can be estimated by classical approaches such as maximum likelihood estimation. Blain et al. [26] and Mercier et al. [36] have dealt in detail with this problem, so we do not repeat it here.

Although our model is developed specially to SCC, it can be extended for other applications. The joint description of degradation and shock phenomena is an example. Indeed, our model belongs to the class of Degradation-Threshold-Shock (DTS) reliability models [42]. In this framework, the Gamma process can still be used to describe the degradation paths, while the Poisson process is applied to express the arrival times of shock events. The classical DTS models assume that the evolutions of degradation and shocks are two independent processes [20, 43, 44]. However, the dependence between them is of importance in many practical situations and should not be neglected in the modeling [45]. From this point of view, some extensions of DTS models considering the dependence between degradation and shock have been developed. The works [46-49] consider that the shock process may cause additional damage for the degradation process, while the works [50, 51] recently consider that the degradation process can drive the arrival rate of shock events. Our model, sharing the same idea of the former works, considers that the degradation process is influenced by shock process. Observing the evolution path of $X_{t}^{S}$, we find that the degradation rate changes instantaneously whenever a shock arrives. Besides, the model also presents two kinds of competing risks. The first competition is between shock process and degradation process to "contribute" to the system failure, the second competition is between the primary crack processes themselves to "contribute" to the failure due to degradation.

\section{Diagnostic and Prognostic Condition Indices}

Diagnosis and prognosis can provide condition indices essential for maintenance decision-making. In this section, four typical condition indices are considered: two diagnostic indices characterizing the partial and complete information about the current system health, and two prognostic indices related to the average and the probability law of the system residual useful life (RUL). The former indices are revealed through inspection operations, while the latter ones are constructed using also the degradation and failure model developed in Section 2. Some properties of the prognostic indices are also analyzed for maintenance decision-making purposes.

\subsection{Diagnostic Condition Indices}

Diagnosis process determines the system health state based on the current monitoring observations [13]. Diagnostic condition indices are the results of such process. In reality, the diagnosis is not a simple task and may require sophisticated techniques (see e.g., $[12,13])$. The present paper does not aim at developing such diagnosis approaches, but rather at 
investigating the ways to incorporate diagnostic condition indices in maintenance decision-making. So, we just simply assume that the diagnosis is attached to inspection operations which can perfectly reveal the current length and number of cracks. Consequently, we can define the three following diagnostic condition indices

- $N_{t}$ - number of cracks up to time $t$,

- $X_{t}^{S}$ - sum of all crack sizes up to time $t$,

- $\left(X_{t}^{S}, N_{t}\right)$ - couple of sum of all crack sizes and number of cracks up to time $t$.

$N_{t}$ cannot completely characterize the current health state of system, while $X_{t}^{S}$ represents the crack sizes at time $t$ directly, and the associated number of cracks indirectly. Compared to the other diagnostic condition indices, $N_{t}$ is obviously not enough to ensure an efficient and robust maintenance decision. This is why we just study here the two diagnostic condition indices $X_{t}^{S}$ and $\left(X_{t}^{S}, N_{t}\right)$, and the associated maintenance strategies.

\subsection{Prognostic Condition Indices}

Prognostic condition indices are the results of the prognosis of future system health. RUL, defined as the length from the current time to the end of the system useful life, is a well-known prognostic information because it can provide an idea about how long a system at a particular age will still survive. The concept of RUL has been widely investigated by many works in the fields of prognosis and CBM [10]. The present paper adds a new dimension to these fields by further considering two phenotypes of RUL: average-based RUL index and distribution-based RUL index. The former is usually given by a non-probabilistic approach (e.g., artificial neural networks, fuzzy logic model, etc.), while the latter may be returned by a probabilistic approach (e.g., stochastic process, bayesian networks, etc.) [52]. They are the two prognostic condition indices that we study in this paper. Indeed, based on [9], given the number of cracks $N_{t}$ and the sum of their crack sizes $X_{t}^{S}$ returned by inspection operations, the system RUL at time $t$ is expressed as

$$
\rho\left(t \mid X_{t}^{S}, N_{t}\right)=Z-t \mid Z>t, X_{t}^{S}, N_{t}
$$

where $Z$ is the system failure time defined by (8). Its survival function is then given by

$$
P\left(\rho\left(t \mid X_{t}^{S}, N_{t}\right)>u\right)=P\left(Z>t+u \mid Z>t, X_{t}^{S}, N_{t}\right)=R\left(t+u \mid X_{t}^{S}, N_{t}\right)
$$

where $R\left(t+u \mid X_{t}^{S}, N_{t}\right)$ denotes the system conditional reliability at time $t+u$ given $X_{t}^{S}$ and $N_{t}$. If $X_{t}^{S}=x<L$ and $N_{t}=k<N$, this reliability is computed as

$$
\begin{aligned}
R(t+u \mid x, k) & =P\left(X_{t+u}^{S}<L, N_{t+u}<N \mid X_{t}^{S}=x, N_{t}=k\right) \\
& =\sum_{h=0}^{N-1-k} P\left(X_{t+u}^{S}<L \mid X_{t}^{S}=x, N_{t}=k, N_{t+u}-N_{t}=h\right) P\left(N_{t+u}-N_{t}=h\right) \\
& =\sum_{h=0}^{N-1-k} P\left(X_{[t, t+u]}^{S}<L-x \mid N_{t}=k, N_{t+u}-N_{t}=h\right) \mathrm{e}^{-\lambda u} \frac{(\lambda u)^{h}}{h !} \\
& =\sum_{h=0}^{N-1-k} \mathrm{e}^{-\lambda u} \frac{(\lambda u)^{h}}{h !} F_{X_{[t, t+u]}^{S} \mid N_{t}, N_{t+u}-N_{t}}(L-x \mid k, h),
\end{aligned}
$$

where $F_{X_{[t, t+u]}^{S} \mid N_{t}, N_{t+u}-N_{t}}(L-x \mid k, h)$ is given by (7). Otherwise, $R(t+u \mid x, k)=0$. Thus, $R\left(t+u \mid X_{t}^{S}, N_{t}\right)$ can be seen as the distribution-based RUL index. From (11), the average-based RUL index (i.e., mean residual life - MRL) at time $t$, $\mu\left(t \mid X_{t}^{S}, N_{t}\right)$, given $X_{t}^{S}=x<L$ and $N_{t}=k<N$ can by computed as follows

$$
\mu(t \mid x, k)=E[\rho(t \mid x, k)]=\int_{0}^{\infty} R(u \mid x, k) d u
$$

Figures $2 \mathrm{a}$ and $2 \mathrm{~b}$ show respectively the shape of the conditional reliability $R(t+u \mid x, k)$ and the conditional MRL $\mu(t \mid x, k)$ of the system when $x$ and $k$ vary. One can remark that $R(t+u \mid x, k)$ and $\mu(t \mid x, k)$ are non-increasing functions of $x$ and/or 


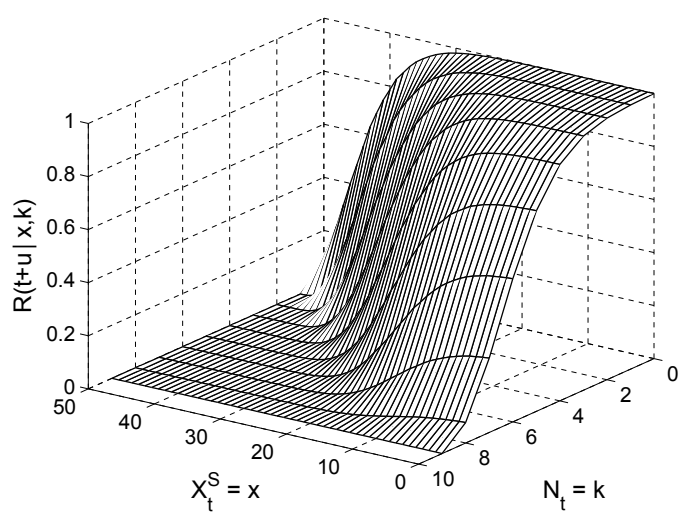

(a) System reliability $R(t+u \mid x, k)-u=5$.

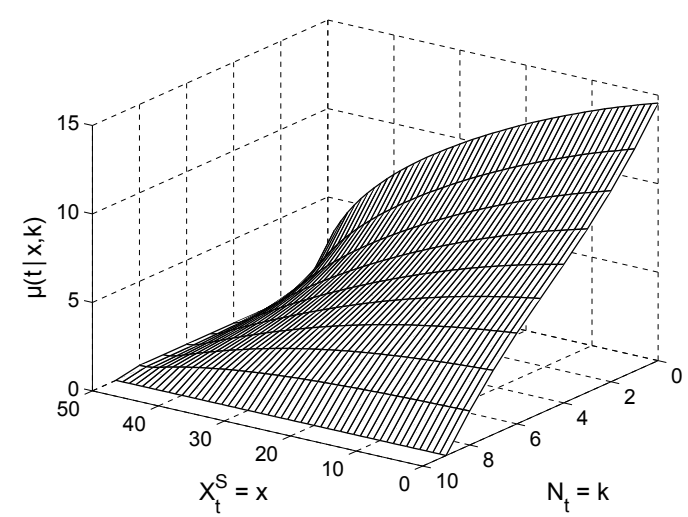

(b) System MRL $\mu(t \mid x, k)$.

Fig. 2 - Shape of the system RUL - $\alpha=1, \beta=1, \lambda=0.5, L=50, N=9$.

and for a fixed $x, 0 \leq x \leq L, R(t+u \mid x, k)$ is non-increasing in $k$. Consequently, for $0 \leq x_{1} \leq x_{2} \leq L$ and $0 \leq k_{1} \leq k_{2} \leq N$, the conditional reliability and the conditional MRL of system satisfy the properties

$$
\begin{gathered}
R\left(t+u \mid x_{1}, k_{1}\right) \geq R\left(t+u \mid x_{2}, k_{1}\right) \geq R\left(t+u \mid x_{2}, k_{2}\right) \geq 0 \text { and } R\left(t+u \mid x_{1}, k_{1}\right) \geq R\left(t+u \mid x_{1}, k_{2}\right) \geq R\left(t+u \mid x_{2}, k_{2}\right) \geq 0 \\
\mu\left(t \mid x_{1}, k_{1}\right) \geq \mu\left(t \mid x_{2}, k_{1}\right) \geq \mu\left(t \mid x_{2}, k_{2}\right) \geq 0 \text { and } \mu\left(t \mid x_{1}, k_{1}\right) \geq \mu\left(t \mid x_{1}, k_{2}\right) \geq \mu\left(t \mid x_{2}, k_{2}\right) \geq 0 .
\end{gathered}
$$

The monotonicity property of the conditional reliability and the conditional MRL of the system is very important for building CBM strategies based on prognostic condition indices and the associated cost models.

\section{Condition-based Maintenance Strategies}

This section aims at showing how the diagnostic and prognostic condition indices considered in Section 3 can be incorporated into the maintenance decision-making. Four CBM strategies corresponding to these indices are then proposed. To avoid upsetting the aforementioned CM strategies, these maintenance strategies still rely on periodic inspection schemes. The proposed illustrations are based on the system characterized by the set of parameters $\alpha=0.5, \beta=0.5, \lambda=1, L=50$ and $N=9$.

\subsection{Maintenance Assumptions}

Considering the system described in Section 2, we assume that the system health state is hidden. This means that the system reveals only the number of cracks, the associated crack sizes, and its failure/working state through an inspection operation. Note that the notion of inspection mentioned here is not simply the data collection, but also the feature extraction from the collected data, the construction of degradation indicators, and perhaps more [53]. In other words, this activity includes all the tasks before the Maintenance Decision Making task in a CBM program [6]. Such an inspection operation is itself costly, and takes time; however, compared to the life cycle of a system, the time for an inspection is negligible. Thus, we assume that each inspection operation is instantaneous, perfect, non-destructive, and incurs a cost $C_{i}$.

Two maintenance actions are available on the system: a preventive replacement (PR) with $\operatorname{cost} C_{p} \geq C_{i}$, and a corrective replacement $(\mathrm{CR})$ with cost $C_{c}$. Since maintenance operations are the true physical replacement such that the system is as-good-as-new rather than repairs, the interventions take negligible times and their costs are fixed, irrespective of the degradation level (i.e., crack length and/or crack number) of the system. Even though both the PR and CR actions put the system back in the as-good-as-new state, they are not necessarily identical in practice because the CR is unplanned and it has to be performed on a more deteriorated system, and the cost $C_{c}$ can also comprise different costs associated to failure like damage to the environment. It is thus likely to be more complex and more expensive (i.e. $C_{c}>C_{p}$ ). Moreover, we assume that a replacement can only be instantaneously performed at predetermined inspection times. Therefore, there exists a system inactivity after failure and an additional cost is incurred from the failure time until the next replacement time at a cost rate $C_{d}$. 
For the above maintenance costs, $C_{i}, C_{p}$ and $C_{c}$ are measured in cost unit, while $C_{d}$ is measured in cost unit/time unit. The cost unit may be U.S. Dollar, Euro, or British Pound, etc., and time unit may be month, quarter, or year, etc.

\subsection{Maintenance Strategies}

The majority of maintenance strategies developed in the literature are of control-limit type [54], and their optimality in a Markovian framework has been shown in [55-58]. Within this class of control-limit rule maintenance strategies, we propose four periodic inspection and replacement strategies: two diagnosis-based strategies called $(T, M)$ and $\left(T, M, N_{p}\right)$, and two prognosis-based strategies called $\left(T, \mu_{p}\right)$ and $\left(T, R_{p}\right)$. The only difference among these strategies is the way to make a PR decision. So, for a compact representation, in the following, we present first the common structure of periodic inspection and replacement strategies, then we make explicit the PR decision for each considered strategy.

\subsubsection{Common structure of periodic inspection and replacement strategies}

Under the framework of periodic inspection and replacement strategies, the system is regularly inspected with period $T$ ( $T$ is measured in time unit). At an inspection date $\tau_{j}=j T, j=1,2, \ldots$, if the systems fails due to an excessive level of sum of crack size (i.e., $X_{\tau_{j}}^{S} \geq L$ ) or due to an excessive number of cracks (i.e., $N_{\tau_{j}} \geq N$ ), it is correctively replaced. But if the system is still running at this date, a decision based on one of above condition indices is made. If the PR condition is satisfied, the system is preventively replaced at $\tau_{j}$. Otherwise, the system is considered healthy, so nothing is done at $\tau_{j}$, and the maintenance decision is postponed, at least, until the next inspection time $\tau_{j+1}=\tau_{j}+T$.

\subsection{2. $(T, M)$ strategy}

This maintenance strategy represents a traditional and naive use of condition indices where only partial information about the current health state of system is integrated in maintenance decision-making. In our present case, this corresponds to the use of the diagnostic condition index $X_{t}^{S}$. Since the growing of the sum of crack size $X_{t}^{S}$ increases the probability of system failure, a threshold $M$ should be introduced to anticipate the system failure. In fact, at an inspection date $\tau_{j}=j T$, $j=1,2, \ldots$, if $M \leq X_{\tau_{j}}^{S}<L$, the running system is considered too degraded, and a PR should be carried out. As such, the inspection period $T$ and PR threshold $M$ are the two decision parameters to be optimized, and we call this strategy ( $T$, $M$ ) to enhance the importance of these parameters.

For an illustration, we apply the $(T, M)$ strategy with the decision parameters $T=2$ and $M=30$ for the system defined as above, then we sketch the evolution of the sum of crack sizes $\left\{X_{\tau_{j}}^{S}\right\}_{j \in \mathbb{N}}$ and the associated numbers of cracks $\left\{N_{\tau_{j}}\right\}_{j \in \mathbb{N}}$ at inspection times. Fig. 3 shows the numerical results. In this figure, as well as in following figures, the notations $Q_{\tau_{j}^{-}}$and $Q_{\tau_{j}^{+}}$refer respectively to the values of the quantity $Q$ at the time just before, and just after the inspection or replacement time $\tau_{j}$ ( $Q$ may be the sum of crack sizes, the number of cracks, the system MRL or the system conditional reliability). We can see clearly that the behavior of the maintained system depends closely on the values of the inspection period $T$ and of the threshold $M$. Although the PR threshold is only applied for the sum of crack sizes, it can still drive the evolution of the number of cracks thanks to the dependence between these two quantities. In other words, besides avoiding the failures due to crack size, the threshold $M$ also helps to prevent the failures due to cracks number indirectly. The sequences of maintenance operations in Fig. 3 is explicitly presented in Table 1.
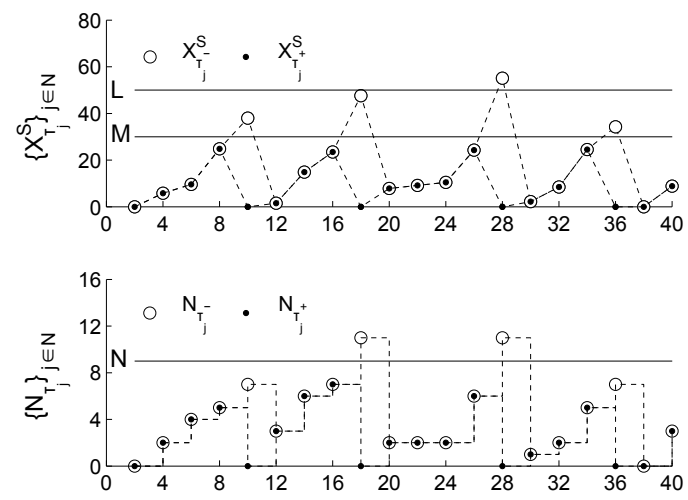

Fig. 3 - Behavior of the maintained system under the $(T, M)$ strategy. 


\begin{tabular}{|c|cccc|}
\hline Time & $5 T$ & $9 T$ & $14 T$ & $18 T$ \\
\hline Operation & $\mathrm{PR}$ & $\mathrm{CR}^{N}$ & $\mathrm{CR}^{X}$ & $\mathrm{PR}$ \\
\hline
\end{tabular}

Table 1 - Sequence of maintenance operations under the $(T, M)$ strategy.

\subsection{3. $\left(T, M, N_{p}\right)$ strategy}

Using solely the threshold $M$ to prevent both kinds of failures due to crack size and cracks number as in the $(T, M)$ strategy might seem suboptimal, as it could better to take also into account the number of cracks. A simple way to improve this is to introduce, along with the threshold $M$, an additional threshold $N_{p}$ for the number of cracks to anticipate separately these failures. Such a strategy allows exploiting the complete information about the current system health (i.e., couple of diagnostic condition indices $\left(X_{t}^{S}, N_{t}\right)$ in our present case) for maintenance decision-making purpose. More precisely, a PR should be carried out at an inspection date $\tau_{j}=j T, j=1,2, \ldots$, if $M \leq X_{\tau_{j}}^{S}<L$ or $N_{p} \leq N_{\tau_{j}}<N$. The inspection period $T$ and the PR thresholds $M$ and $N_{p}$ are the three decision parameters to be optimized, and thus this strategy is called $\left(T, M, N_{p}\right)$.
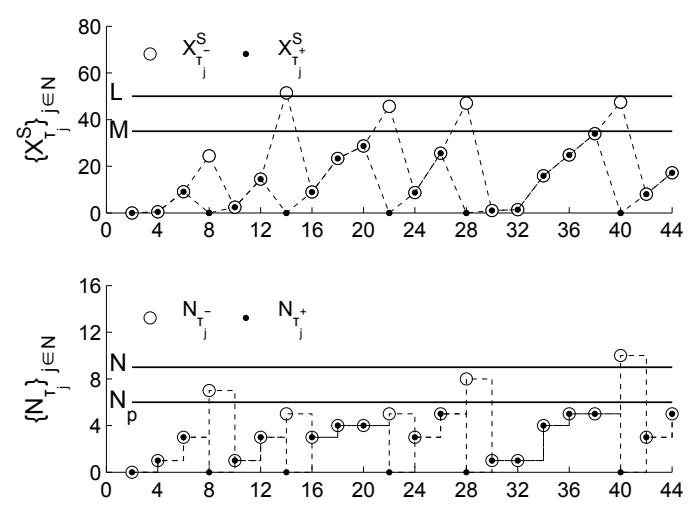

Fig. 4 - Behavior of the maintained system under the $\left(T, M, N_{p}\right)$ strategy.

To illustrate the $\left(T, M, N_{p}\right)$ strategy, we chose decision parameters as $T=2, M=30$ and $N_{p}=6$. The evolution of the sum of crack sizes $\left\{X_{\tau_{j}}^{S}\right\}_{j \in \mathbb{N}}$ and the associated number of cracks $\left\{N_{\tau_{j}}\right\}_{j \in \mathbb{N}}$ under the $\left(T, M, N_{p}\right)$ strategy are sketched in Fig. 4. Obviously, the use of both thresholds $M$ and $N_{p}$ allows more flexibility in PR decision-making: the $\left(T, M, N_{p}\right)$ strategy returns to the $(T, M)$ strategy when $N_{p} \equiv N$. This is why the former can lead to more cost saving than the latter. Table 2 explicitly presents the sequences of maintenance operations in Fig. 4.

\begin{tabular}{|c|ccccc|}
\hline Time & $4 T$ & $7 T$ & $11 T$ & $14 T$ & $20 T$ \\
\hline Operation & $\mathrm{PR}^{N}$ & $\mathrm{CR}^{X}$ & $\mathrm{PR}^{X}$ & $\mathrm{PR}^{X}$ or $\mathrm{PR}^{N}$ & $\mathrm{CR}^{N}$ \\
\hline
\end{tabular}

Table 2 - Sequence of maintenance operations under the $\left(T, M, N_{p}\right)$ strategy.

\subsection{4. $\left(T, \mu_{p}\right)$ strategy}

Unlike the above strategies whose PR decisions are based on diagnostic condition indices, we use here an average-based RUL prognostic index of the future system health to provide a decision. More precisely, the MRL of the system is taken into account. Since the value of MRL is always decreasing and reaches 0 when the system fails, to anticipate the system failure, we should introduce a threshold on the MRL (denoted $\mu_{p}$ ) below which a PR operation is carried out. Based on this idea, Huynh et al. have build in [23] a predictive maintenance strategy for a so-called DTDS model. A similar strategy is considered in this paper. Indeed, if the system is still running at an inspection time $\tau_{j}=j T, j=1,2, \ldots$, , given the detected number of cracks $N_{\tau_{j}}$ and the sum of all appeared crack sizes $X_{\tau_{j}}^{S}$, we compute the system MRL, $\mu\left(\tau_{j} \mid X_{\tau_{j}}^{S}, N_{\tau_{j}}\right)$, according to (12). If $0<\mu\left(\tau_{j} \mid X_{\tau_{j}}^{S}, N_{\tau_{j}}\right)<\mu_{p}$, the running system is considered too degraded and should be preventively replaced at $\tau_{j}$. The inspection period $T$ and the $\mathrm{PR}$ threshold $\mu_{p}$ are the two decision parameters to be optimized, and thus this strategy is called $\left(T, \mu_{p}\right)$.

Applying the $\left(T, \mu_{p}\right)$ strategy with decision parameters $T=2$ and $\mu_{p}=3$ for the considered system, we obtain its behavior as in Fig. 5. Fig. 5a shows the evolution of the sum of crack sizes $\left\{X_{\tau_{j}}^{S}\right\}_{j \in \mathbb{N}}$ and the associated number of cracks $\left\{N_{\tau_{j}}\right\}_{j \in \mathbb{N}}$, 

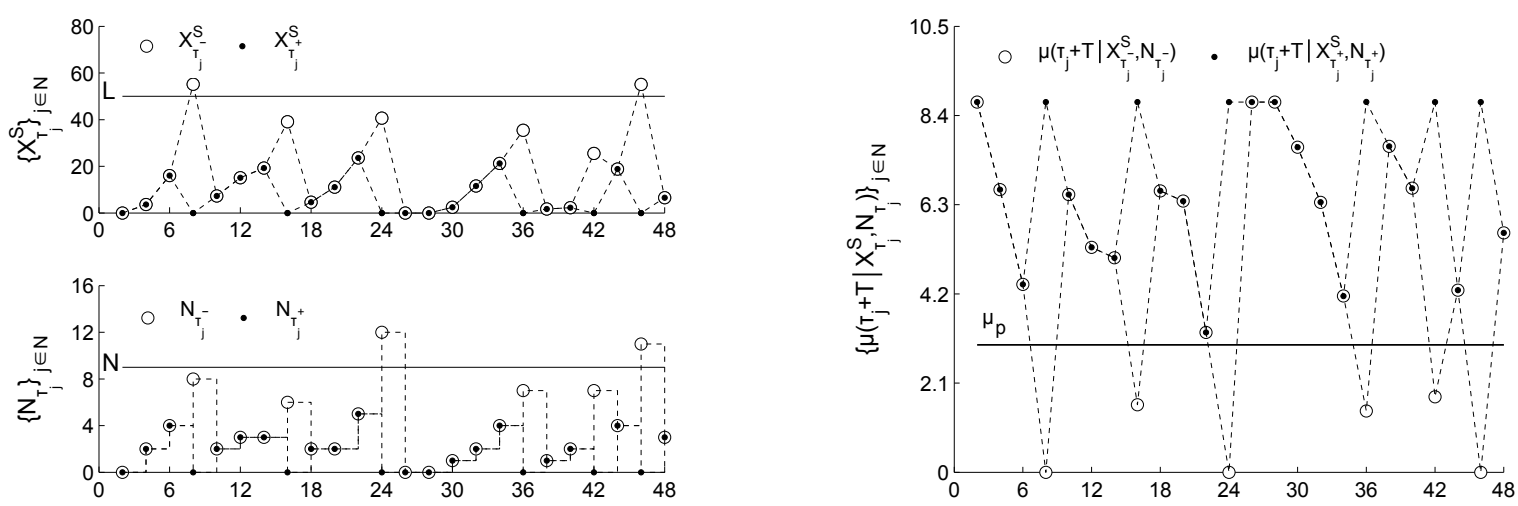

(a) Evolution of $X_{\tau_{j}}^{S}$ and $N_{\tau_{j}}$

(b) Evolution of $\mu\left(\tau_{j} \mid X_{\tau_{j}}^{S}, N_{\tau_{j}}\right)$

Fig. 5 - Behavior of the maintained system under the $\left(T, \mu_{p}\right)$ strategy.

while Fig. 5b represents the evolution of system MRL $\left\{\mu\left(\tau_{j} \mid X_{\tau_{j}}^{S}, N_{\tau_{j}}\right)\right\}_{j \in \mathbb{N}}$ under the $\left(T, \mu_{p}\right)$ strategy. Indirectly, the behavior of the maintained system is driven by the value of the PR threshold $\mu_{p}$. Indeed, through $\mu_{p}$, the $\left(T, \mu_{p}\right)$ strategy also allows incorporating the complete information about the current system health into maintenance decision as the $\left(T, M, N_{p}\right)$ strategy. What is more, only two instead of three decision parameters have to be controlled in the $\left(T, \mu_{p}\right)$ strategy, the maintenance decision-making and the parameters optimization in the former are hence easier than in the latter. These are some advantages of using the system MRL instead of diagnostic condition indices. The sequences of maintenance operations in Fig. 5 are explicitly presented in Table 3.

\begin{tabular}{|c|cccccc|}
\hline Time & $8 T$ & $16 T$ & $24 T$ & $36 T$ & $42 T$ & $46 T$ \\
\hline Operation & $\mathrm{CR}^{X}$ & $\mathrm{PR}$ & $\mathrm{CR}^{N}$ & $\mathrm{PR}$ & $\mathrm{PR}$ & $\mathrm{CR}^{X}$ or $\mathrm{CR}^{N}$ \\
\hline
\end{tabular}

Table 3 - Sequence of maintenance operations under the $\left(T, \mu_{p}\right)$ strategy.

\subsection{5. $\left(T, R_{p}\right)$ strategy}

Through this strategy, we aim at showing a way to incorporate a distribution-based RUL prognosis index of the future system health into maintenance decision-making. To do so, we base the maintenance decisions on the system conditional reliability and its monotony (see also the end of Section 3). Indeed, a threshold $R_{p}$ is introduced, and whenever the value of the system conditional reliability at an inspection time is lower than $R_{p}$, PR operations are done. More in detail, if the system running at an inspection date $\tau_{j}=j T, j=1,2, \ldots$, given the detected number of cracks $N_{\tau_{j}}$ and the sum of all appeared crack sizes $X_{\tau_{j}}^{S}$, we compute the system conditional reliability at the next inspection time, $R\left(\tau_{j}+T \mid X_{\tau_{j}}^{S}, N_{\tau_{j}}\right)$, according to (11) and we trigger a $\mathrm{PR}$ at the current inspection date if $0<R\left(\tau_{j}+T \mid X_{\tau_{j}}^{S}, N_{\tau_{j}}\right)<R_{p}$. The inspection period $T$ and the PR threshold $R_{p}$ are the two decision parameters to be optimized, and this strategy is hence called ( $\left.T, R_{p}\right)$.

For an illustration, we apply the $\left(T, R_{p}\right)$ strategy with decision parameters $T=2$ and $R_{p}=0.45$ for the considered system, and we sketch its behavior. Fig. 6a shows the evolution of the sum of crack sizes $\left\{X_{\tau_{j}}^{S}\right\}_{j \in \mathbb{N}}$ and the number of cracks $\left\{N_{\tau_{j}}\right\}_{j \in \mathbb{N}}$, and Fig. 6b represents the associated evolution of system conditional reliability $\left\{R\left(\tau_{j}+T \mid X_{\tau_{j}}^{S}, N_{\tau_{j}}\right)\right\}_{j \in \mathbb{N}}$ Table 4 explicitly presents the sequences of maintenance operations in Fig. 6. One can remark that, as the system MRL,

\begin{tabular}{|c|ccccc|}
\hline Time & $14 T$ & $24 T$ & $30 T$ & $40 T$ & $48 T$ \\
\hline Operation & $\mathrm{CR}^{N}$ & $\mathrm{CR}^{X}$ & $\mathrm{PR}$ & $\mathrm{PR}$ & $\mathrm{PR}$ \\
\hline
\end{tabular}

Table 4 - Sequence of maintenance operations under the $\left(T, R_{p}\right)$ strategy.

the system conditional reliability contains the complete information about the current system health.

\section{Maintenance Cost Models, and Optimization}

To have a precise analysis on the performances and robustness of the four maintenance strategies considered in Section 4, we should rely on their mathematical cost models. This is the main aim of this section. For the illustrations of the cost 

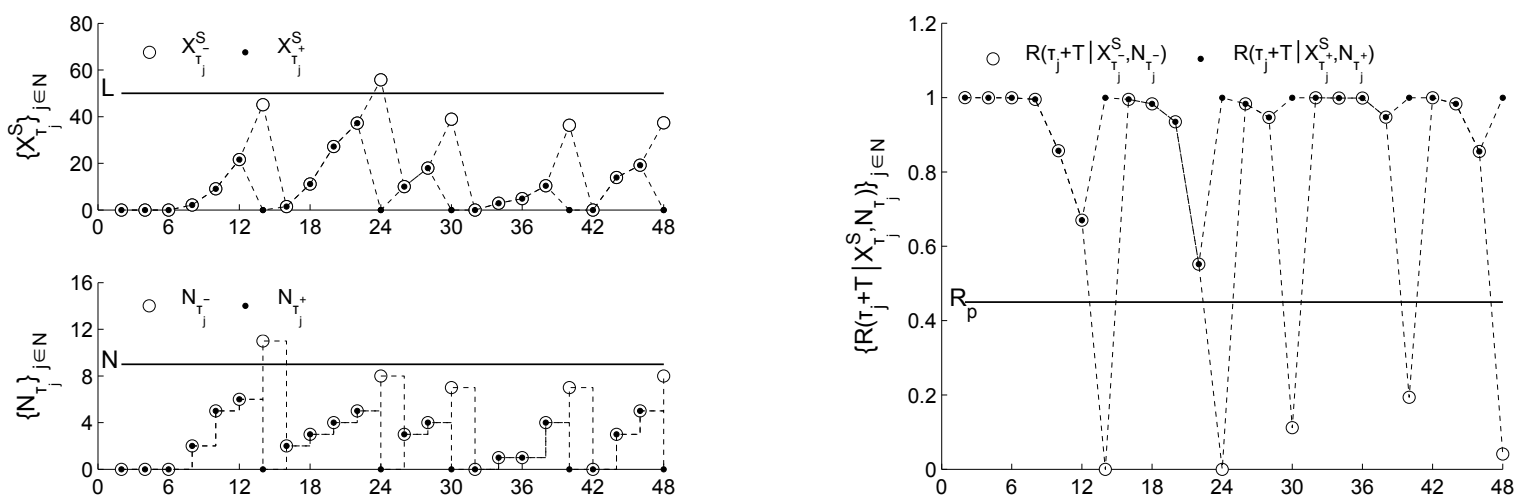

(a) Evolution of $X_{\tau_{j}}^{S}$ and $N_{\tau_{j}}$.

(b) Evolution of $R\left(\tau_{j}+T \mid X_{\tau_{j}}^{S}, N_{\tau_{j}}\right)$.

Fig. 6 - Behavior of the maintained system under the $\left(T, R_{p}\right)$ strategy.

models developed within this section, the system is defined by the set of parameters $\alpha=1, \beta=1, \lambda=0.5, L=50$ and $N=9$, and the used set of maintenance costs is $C_{i}=5, C_{p}=50, C_{c}=100$ and $C_{d}=25$.

\subsection{Cost Criterion, and General Cost Model of Periodic Inspection and Replacement Strategies}

To assess the performance and robustness of the considered maintenance strategies, we focus on a widely used criterion which is the long-run expected maintenance cost rate $C_{\infty}$ defined as [17]

$$
C_{\infty}=\lim _{t \rightarrow \infty} \frac{E[C(t)]}{t}
$$

where $C(t)$ is the total maintenance cost including the downtime cost up to time $t$. $C_{\infty}$ is measured in cost unit/time unit. Under the considered maintenance strategies, after an as-good-as-new (preventive or corrective) replacement, the system starts again from the new state and evolves independently from the past (i.e., the history and past events of the system before the replacement) with the same probabilistic behavior. The evolution of the maintained system can be hence assimilated to a regenerative process in which renewal points are replacement times. Thus, we can apply the well-known renewal-reward theorem to compute (13) as the ratio of the expected maintenance cost on the first renewal cycle over the expected length of the first cycle [28, page 42$]$

$$
C_{\infty}=\frac{E\left[C\left(S_{1}\right)\right]}{E\left[S_{1}\right]}
$$

where $S_{1}$ denotes the first replacement time of the system.

Now, we give a general framework to compute the long-run expected cost rate of periodic inspection and replacement strategies. Since a replacement, whether preventive or corrective, is only performed at an inspection date, we can assume, without loss of generality, that the system is first replaced at $S_{1}=k T, k=1,2, \ldots$. The cumulative maintenance cost at $S_{1}$ consist of the cost of $k$ inspections, a PR cost or a CR cost, and downtime cost if the maintained system fails at a time $Y \in[(k-1) T, k T]$. Thus, the expected maintenance cost accumulated in a renewal cycle is computed as

$$
E\left[C\left(S_{1}\right)\right]=\sum_{k=1}^{\infty}\left\{\left(C_{p}+k C_{i}\right) P_{p, k}(k T)+\left(C_{c}+k C_{i}\right) P_{c, k}(k T)+C_{d} E\left[W_{d, k}(k T)\right]\right\}
$$

where $E\left[W_{d, k}(k T)\right]$ denotes the expected downtime in a renewal cycle, which can be obtained by

$$
E\left[W_{d, k}(k T)\right]=E[k T-Y]=E\left[\int_{(k-1) T}^{k T} 1_{\{(k-1) T<Y<t\}} d t\right]=\int_{(k-1) T}^{k T} P((k-1) T<Y<t) d t
$$

$P_{p, k}(k T)$ and $P_{c, k}(k T)$ represent respectively the probabilities of $\mathrm{PR}$ and $\mathrm{CR}$ at time $k T$. The probability $P_{c, k}(k T)$ can be explained through the variable $Y$ as

$$
P_{c, k}(k T)=P((k-1) T<Y<k T) .
$$


The expected length of the first renewal cycle is given by

$$
E\left[S_{1}\right]=\sum_{k=1}^{\infty}\left\{k T\left(P_{p, k}(k T)+P_{c, k}(k T)\right)\right\}
$$

As such, the long-run expected cost rate of any periodic inspection and replacement strategies can be completely evaluated by

$$
C_{\infty}=\frac{\sum_{k=1}^{\infty}\left\{\left(C_{p}+k C_{i}\right) P_{p, k}(k T)+\left(C_{c}+k C_{i}\right) P_{c, k}(k T)+C_{d} E\left[W_{d, k}(k T)\right]\right\}}{\sum_{k=1}^{\infty}\left\{k T\left(P_{p, k}(k T)+P_{c, k}(k T)\right)\right\}}
$$

if $P_{p, k}(k T)$ and $P((k-1) T<Y<t)$ are known. Evidently, the values of these two probabilities closely depend on the used maintenance strategies and on the value of the maintenance decision variables in each of them. As such, the cost $C_{\infty}$ depends on the maintenance decision variables through $P_{p, k}(k T)$ and $P((k-1) T<Y<t)$. In the following, we focus on formulating these quantities according to the four proposed strategies.

\subsection{Maintenance Cost Model of The $(T, M)$ Strategy}

The long-run expected maintenance cost rate of the $(T, M)$ strategy, $C_{\infty}^{(T, M)}$, is obtained from (19), where the probabilities $P_{p, k}^{(T, M)}(k T)$ and $P^{(T, M)}((k-1) T<Y<t)$ are formulated as follows.

According to the $(T, M)$ strategy, a PR is triggered if the sum of crack sizes of the running system belongs to the interval $[M, L[$. Its probability at time $k T$ is therefore computed as

$$
\begin{aligned}
& P_{p, k}^{(T, M)}(k T)=P\left(X_{(k-1) T}^{S}<M \leq X_{k T}^{S}<L, N_{(k-1) T} \leq N_{k T}<N\right)= \\
& \sum_{n=0}^{N-1} \mathrm{e}^{-\lambda(k-1) T} \frac{(\lambda(k-1) T)^{n}}{n !} \int_{0}^{M} \sum_{m=0}^{N-1-n} \frac{(\lambda T)^{m}}{m !} \mathrm{e}^{-\lambda T}\left(F_{X_{[(k-1) T, k T]}^{S} \mid N_{(k-1) T}, N_{k T}-N_{(k-1) T}}(L-x \mid n, m)-\right. \\
& \left.F_{X_{[(k-1) T, k T]}^{S} \mid N_{(k-1) T}, N_{k T}-N_{(k-1) T}}(M-x \mid n, m)\right) f_{X_{(k-1) T}^{S} \mid N_{(k-1) T}}(x \mid n) d x
\end{aligned}
$$

where $f_{X_{(k-1) T}^{S} \mid N_{(k-1) T}}(\cdot)$ and $F_{X_{[(k-1) T, k T]}^{S} \mid N_{(k-1) T}, N_{k T}-N_{(k-1) T}}(\cdot)$ are given by (6) and (7) respectively.

The probability that the system fails in the time interval $[(k-1) T, t]$ under the $(T, M)$ strategy is given by

$$
P^{(T, M)}((k-1) T<Y<t)=P\left(X_{(k-1) T}^{S}<M, N_{(k-1) T}<N,\left(X_{t}^{S} \geq L \text { or } N_{t} \geq N\right)\right)=P_{Y, 1}^{(T, M)}+P_{Y, 2}^{(T, M)}
$$

where $P_{Y, 1}^{(T, M)}$ and $P_{Y, 2}^{(T, M)}$ are expressed as

$$
\begin{aligned}
& P_{Y, 1}^{(T, M)}= P\left(X_{(k-1) T}^{S}<M, N_{(k-1) T}<N \leq N_{t}\right)= \\
& \sum_{n=0}^{N-1} \mathrm{e}^{-\lambda(k-1) T} \frac{(\lambda(k-1) T)^{n}}{n !}\left(1-\sum_{m=0}^{N-1-n} \mathrm{e}^{-\lambda(t-(k-1) T)} \frac{(\lambda(t-(k-1) T))^{m}}{m !}\right) F_{X_{(k-1) T}^{S} \mid N_{(k-1) T}}(M \mid n), \\
& P_{Y, 2}^{(T, M)}=P\left(X_{(k-1) T}^{S}<M<L \leq X_{t}^{S}, N_{(k-1) T}<N_{t}<N\right)= \frac{(\lambda(k-1) T)^{n}}{n !} \int_{0}^{M-1} \sum_{m=0}^{N-1-n} \mathrm{e}^{-\lambda(t-(k-1) T)} \frac{(\lambda(t-(k-1) T))^{m}}{\left(1-F_{X_{[(k-1) T, t]}^{S} \mid N_{(k-1) T}, N_{t}-N_{(k-1) T}}(L-x \mid n, m)\right) f_{X_{(k-1) T}^{S} \mid N_{(k-1) T}}(x \mid n) d x} \\
& \sum_{n=0}^{N-\lambda} \mathrm{e}^{-\lambda(k-1) T} \frac{(L)}{m !}
\end{aligned}
$$

in which $F_{X_{(k-1) T}^{S} \mid N_{(k-1) T}}(\cdot), f_{X_{(k-1) T}^{S} \mid N_{(k-1) T}}(\cdot)$ and $F_{X_{[(k-1) T, t]}^{S} \mid N_{(k-1) T}, N_{t}-N_{(k-1) T}}(\cdot)$ are given by $(5),(6)$ and $(7)$ respectively.

Optimizing the $(T, M)$ strategy amounts to finding the couple of decision parameters $\left(T_{o p t}, M_{o p t}\right)$ which minimizes $C_{\infty}^{(T, M)}$

$$
C_{\infty}^{(T, M)}\left(T_{\text {opt }}, M_{\text {opt }}\right)=\min _{T>0,0<M<L}\left\{C_{\infty}^{(T, M)}(T, M)\right\}
$$

Generalized pattern search algorithms representing in [59] can be used for the optimization purpose. Fig. 7 shows the surface of cost rate of the $(T, M)$ strategy as a function of $T$ and $M$ for the considered system. The form of the cost rate surface 
justifies the existence of a global minimum, and hence finding optimal decision parameters is needed to obtain the best performance of the maintenance strategy. As for the considered system, the $(T, M)$ strategy reaches the minimal cost rate $C_{\infty}^{(T, M)}\left(T_{o p t}, M_{o p t}\right)=6.0339$ at $T_{o p t}=4.6$ and $M_{o p t}=19$.

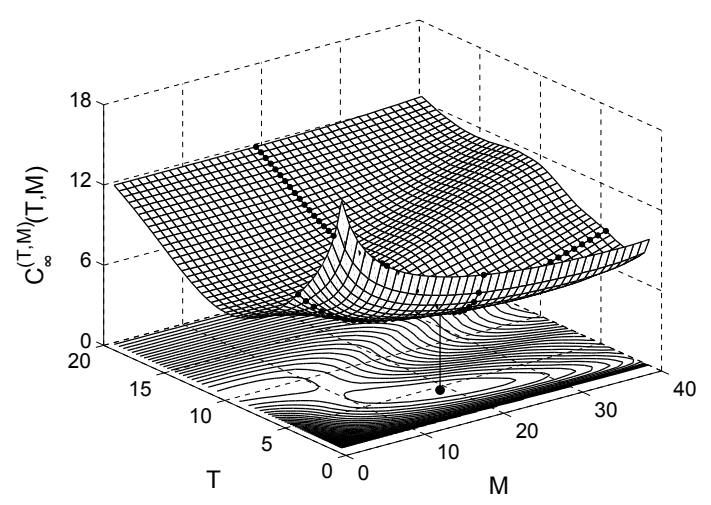

Fig. 7 - Cost rate surface of the $(T, M)$ strategy.

\subsection{Maintenance Cost Model of The $\left(T, M, N_{p}\right)$ Strategy}

The long-run expected maintenance cost rate of the $\left(T, M, N_{p}\right)$ strategy, $C_{\infty}^{\left(T, M, N_{p}\right)}$, is obtained from (19), where the probabilities $P_{p, k}^{\left(T, M, N_{p}\right)}(k T)$ and $P^{\left(T, M, N_{p}\right)}((k-1) T<Y<t)$ are computed as follows.

For the $\left(T, M, N_{p}\right)$ strategy, a PR is carried out when the sum of crack sizes of the running system or the associated number of cracks belongs to the interval $[M, L)$ and $\left[N_{p}, N\right)$ respectively. Its probability at time $k T$ is thus given as

$$
P_{p, k}^{\left(T, M, N_{p}\right)}(k T)=P\left(X_{(k-1) T}^{S}<M, N_{(k-1) T}<N_{p},\left(M \leq X_{k T}^{S}<L \text { or } N_{p} \leq N_{k T}<N\right)\right)=P_{p, k, 1}^{\left(T, M, N_{p}\right)}+P_{p, k, 2}^{\left(T, M, N_{p}\right)},
$$

where $P_{p, k, 1}^{\left(T, M, N_{p}\right)}$ and $P_{p, k, 1}^{\left(T, M, N_{p}\right)}$ are calculated as

$$
\begin{gathered}
P_{p, k, 1}^{\left(T, M, N_{p}\right)}=P\left(X_{(k-1) T}^{S}<M, X_{k T}^{S}<L, N_{(k-1) T}<N_{p} \leq N_{k T}<N\right)= \\
\sum_{n=0}^{N_{p}-1} \frac{(\lambda(k-1) T)^{n}}{n !} \mathrm{e}^{-\lambda(k-1) T} \int_{0}^{M} \sum_{m=N_{p}-n}^{N-1-n} \frac{(\lambda T)^{m}}{m !} \mathrm{e}^{-\lambda T} \times \\
F_{X_{[(k-1) T, k T]}^{S} \mid N_{(k-1) T}, N_{k T}-N_{(k-1) T}}(L-x \mid n, m) f_{X_{(k-1) T}^{S} \mid N_{(k-1) T}}(x \mid n) d x, \\
P_{p, k, 2}^{\left(T, M, N_{p}\right)}=P\left(X_{(k-1) T}^{S}<M \leq X_{k T}^{S}<L, N_{(k-1) T} \leq N_{k T}<N_{p}\right)= \\
\sum_{n=0}^{N_{p}-1} \frac{(\lambda(k-1) T)^{n}}{n !} \mathrm{e}^{-\lambda(k-1) T} \int_{0}^{M} \sum_{m=0}^{N_{p}-1-n} \frac{(\lambda T)^{m}}{m !} \mathrm{e}^{-\lambda T}\left(F_{\left.X_{[(k-1) T}^{S}, k T\right]} \mid N_{(k-1) T}, N_{k T}-N_{(k-1) T}(L-x \mid n, m)-\right. \\
\left.F_{X_{[(k-1) T, k T]}^{S} \mid N_{(k-1) T}, N_{k T}-N_{(k-1) T}}(M-x \mid n, m)\right) f_{X_{(k-1) T}^{S} \mid N_{(k-1) T}}(x \mid n) d x,
\end{gathered}
$$

where $f_{X_{(k-1) T}^{S} \mid N_{(k-1) T}}(\cdot)$ and $F_{X_{[(k-1) T, k T]}^{S} \mid N_{(k-1) T}, N_{k T}-N_{(k-1) T}}(\cdot)$ are given by (6) and (7) respectively.

The probability that the system fails in the time interval $[(k-1) T, t]$ under the $\left(T, M, N_{p}\right)$ strategy is given by

$$
P^{\left(T, M, N_{p}\right)}((k-1) T<Y<t)=P\left(X_{(k-1) T}^{S}<M, N_{(k-1) T}<N_{p},\left(X_{t}^{S} \geq L \text { or } N_{t} \geq N\right)\right)=P_{Y, 1}^{\left(T, M, N_{p}\right)}+P_{Y, 2}^{\left(T, M, N_{p}\right)},
$$

where $P_{Y, 1}^{\left(T, M, N_{p}\right)}$ and $P_{Y, 2}^{\left(T, M, N_{p}\right)}$ are expressed as

$$
\begin{aligned}
P_{Y, 1}^{\left(T, M, N_{p}\right)}= & P\left(X_{(k-1) T}^{S}<M, N_{(k-1) T}<N_{p}, N_{t} \geq N\right)= \\
& \sum_{n=0}^{N_{p}-1} \mathrm{e}^{-\lambda(k-1) T} \frac{(\lambda(k-1) T)^{n}}{n !}\left(1-\sum_{m=0}^{N-1-n} \mathrm{e}^{-\lambda(t-(k-1) T)} \frac{(\lambda(t-(k-1) T))^{m}}{m !}\right) F_{X_{(k-1) T}^{S} \mid N_{(k-1) T}}(M \mid n),
\end{aligned}
$$




$$
\begin{aligned}
& P_{Y, 2}^{\left(T, M, N_{p}\right)}=P\left(X_{(k-1) T}^{S}<M<L \leq\right.\left.X_{t}^{S}, N_{(k-1) T}<N_{p}, N_{t}<N\right)= \\
& \sum_{n=0}^{N_{p}-1} \mathrm{e}^{-\lambda(k-1) T} \frac{(\lambda(k-1) T)^{n}}{n !} \int_{0}^{M} \sum_{m=0}^{N-1-n} \mathrm{e}^{-\lambda(t-(k-1) T)} \frac{(\lambda(t-(k-1) T))^{m}}{m !} \times \\
& \quad\left(1-F_{X_{[(k-1) T, t]}^{S} \mid N_{(k-1) T}, N_{t}-N_{(k-1) T}}(L-x \mid n, m)\right) f_{X_{(k-1) T}^{S} \mid N_{(k-1) T}(x \mid n) d x,}
\end{aligned}
$$

in which $F_{X_{(k-1) T}^{S} \mid N_{(k-1) T}}(\cdot), f_{X_{(k-1) T}^{S} \mid N_{(k-1) T}}(\cdot)$ and $F_{X_{[(k-1) T, t]}^{S} \mid N_{(k-1) T}, N_{t}-N_{(k-1) T}}(\cdot)$ are given by (5), (6) and (7) respectively.

Optimizing the $\left(T, M, N_{p}\right)$ strategy is to find the set of decision parameters $\left(T_{\text {opt }}, M_{\text {opt }}, N_{p, \text { opt }}\right)$ which minimizes $C_{\infty}^{\left(T, M, N_{p}\right)}$

$$
C_{\infty}^{\left(T, M, N_{p}\right)}\left(T_{\text {opt }}, M_{\text {opt }}, N_{p, o p t}\right)=\min _{T>0,0<M<L, 0<N_{p}<N}\left\{C_{\infty}^{\left(T, M, N_{p}\right)}(T, M)\right\} .
$$

In reality, we apply the generalized pattern search algorithms [59] to determine the optimum. Fig. 8 shows the shape of the cost rate of the $\left(T, M, N_{p}\right)$ strategy for the considered system. We obtain the minimal cost rate $C_{\infty}^{\left(T, M, N_{p}\right)}\left(T_{\text {opt }}, M_{\text {opt }}, N_{p, o p t}\right)=$ 5.785 at $T_{o p t}=5, M_{o p t}=23$ and $N_{p, o p t}=5$.

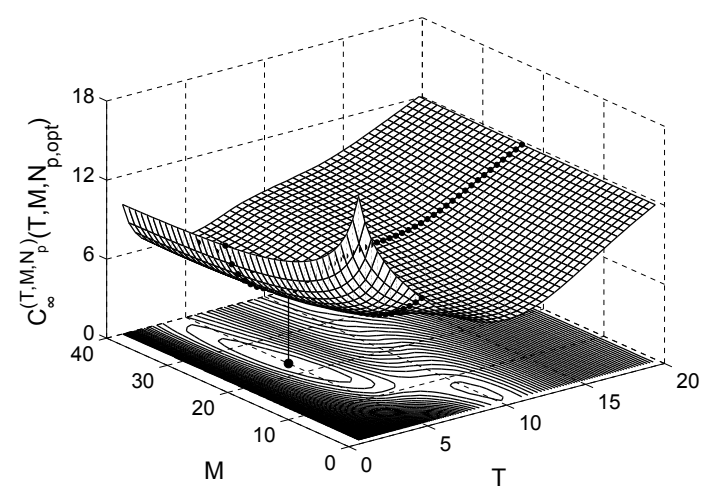

(a) Surface of $C_{\infty}^{\left(T, M, N_{p}\right)}$ when $N_{p}$ is fixed at $N_{p, o p t}=5$.

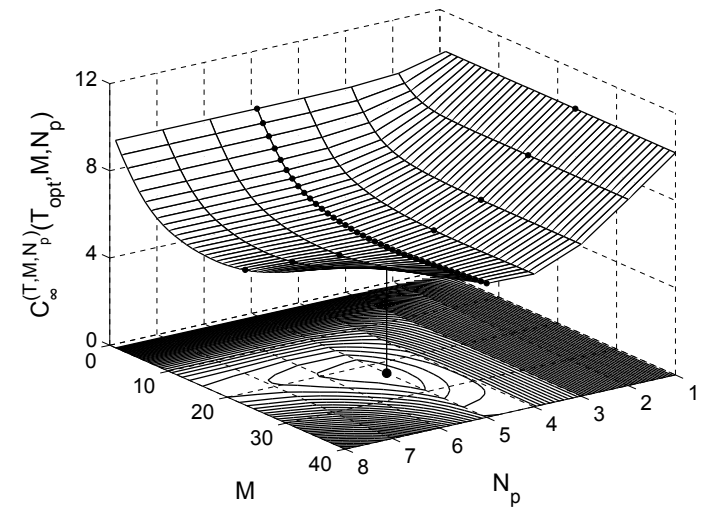

(b) Surface of $C_{\infty}^{\left(T, M, N_{p}\right)}$ when $T$ is fixed at $T_{o p t}=5$.

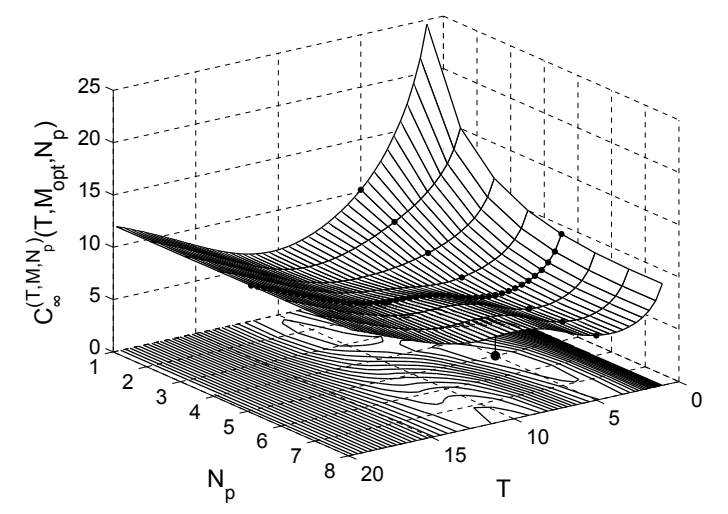

(c) Surface of $C_{\infty}^{\left(T, M, N_{p}\right)}$ when $M$ is fixed at $M_{o p t}=23$.

Fig. 8 - Shape of the cost rate of the $\left(T, M, N_{p}\right)$ strategy.

\subsection{Maintenance Cost Models of The $\left(T, \mu_{p}\right)$ Strategy and The $\left(T, R_{p}\right)$ Strategy}

This section aims at computing the long-run expected maintenance cost rate of the $\left(T, \mu_{p}\right)$ strategy and the $\left(T, R_{p}\right)$ strategy. We will find that the cost rate formulae of both these strategies are the same, despite of their different condition indices used. This is not very surprising because the criterion of interest is the long-run expected maintenance cost rate (13), and from this average viewpoint, the system MRL contains all the information about the system RUL. Consequently, the $\left(T, \mu_{p}\right)$ strategy and the $\left(T, R_{p}\right)$ strategy share the same cost model. For a more compact presentation, $\eta\left(X_{t}^{S}, N_{t}\right)$ and $\eta_{p}$ are used to denote either $\mu\left(t \mid X_{t}^{S}, N_{t}\right)$ or $R\left(t+T \mid X_{t}^{S}, N_{t}\right)$ and either $\mu_{p}$ or $R_{p}$ respectively, and the label $R U L$ is used to represent both strategies name. In the following, we focus on formulating the quantities $P_{p, k}^{R U L}(k T)$ and $P^{R U L}((k-1) T<Y<t)$. The idea is, firstly, to present all the possible events affecting $\eta\left(X_{t}^{S}, N_{t}\right)$ related to each quantity, then to transform these events into the events of sums of crack sizes $X_{t}^{S}$ thanks to the monotonicity property of $\eta\left(X_{t}^{S}, N_{t}\right)$ (review Section 3 ), and 
finally, to compute the associated probabilities. As such, we must define a threshold of crack size corresponding to $\eta_{p}$ as follows. For a fixed number of appeared cracks $k, M_{k}$ is the minimum value of sums of crack sizes $x$ such that the value $\eta(x, k)$ is less than $\eta_{p}$, that is

$$
M_{k}=\inf \left\{x \geq 0, \eta(x, k) \leq \eta_{p}\right\}
$$

If $M_{k}>L$, we impose $M_{k} \equiv L$. We can see that the value of $M_{k}$ is not constant, but adaptive to the appeared cracks number $k$. Using the above idea, the PR probability $P_{p, k}^{R U L}(k T)$ is computed as

$$
\begin{gathered}
P_{p, k}^{R U L}(k T)=P\left(0<\eta\left(X_{k T}^{S}, N_{k T}\right) \leq \eta_{p}<\eta\left(X_{(k-1) T}^{S}, N_{(k-1) T}\right)\right)= \\
P\left(N_{(k-1) T} \leq N_{k T}<N, X_{(k-1) T}^{S}<M_{N_{(k-1) T},}, M_{N_{k T}} \leq X_{k T}^{S}<L\right) .
\end{gathered}
$$

Hence,

$$
\begin{array}{r}
P_{p, k}^{R U L}(k T)=\sum_{n=0}^{N-1} \frac{(\lambda(k-1) T)^{n}}{n !} \mathrm{e}^{-\lambda(k-1) T} \int_{0}^{M_{n}} \sum_{m=0}^{N-1-n} \frac{(\lambda T)^{m}}{m !} \mathrm{e}^{-\lambda T}\left(F_{X_{[(k-1) T, k T]}^{S} \mid N_{(k-1) T}, N_{k T}-N_{(k-1) T}}(L-x \mid n, m)-\right. \\
\left.F_{X_{[(k-1) T, k T]}^{S} \mid N_{(k-1) T}, N_{k T}-N_{(k-1) T}}\left(M_{n+m}-x \mid n, m\right)\right) f_{X_{(k-1) T}^{S} \mid N_{(k-1) T}}(x \mid n) d x,
\end{array}
$$

where $f_{X_{(k-1) T}^{S} \mid N_{(k-1) T}}(\cdot)$ and $F_{X_{[(k-1) T, k T]}^{S} \mid N_{(k-1) T}, N_{k T}-N_{(k-1) T}}(\cdot)$ are given by $(6)$ and $(7)$ respectively.

The probability that the system fails in the time interval $[(k-1) T, t]$ under the $\left(T, \mu_{p}\right)$ strategy and the $\left(T, R_{p}\right)$ strategy is given by

$$
\begin{aligned}
& P^{R U L}((k-1) T<Y<t)=P\left(\eta\left(X_{(k-1) T}^{S}, N_{(k-1) T}\right)>\eta_{p},\left(X_{t}^{S} \geq L \text { or } N_{t} \geq N\right)\right)= \\
& P\left(N_{(k-1) T}<N, X_{(k-1) T}^{S}<M_{N_{(k-1) T}},\left(X_{t}^{S} \geq L \text { or } N_{t} \geq N\right)\right)=P_{Y, 1}^{R U L}+P_{Y, 2}^{R U L},
\end{aligned}
$$

where $P_{Y, 1}^{R U L}$ and $P_{Y, 2}^{R U L}$ are respectively calculated as

$$
\begin{aligned}
& P_{Y, 1}^{R U L}=P\left(N_{(k-1) T}<N \leq N_{t}, X_{(k-1) T}^{S}<M_{N_{(k-1) T}}\right)= \\
& \sum_{n=0}^{N-1} \mathrm{e}^{-\lambda(k-1) T} \frac{(\lambda(k-1) T)^{n}}{n !}\left(1-\sum_{m=0}^{N-1-n} \mathrm{e}^{-\lambda(t-(k-1) T)} \frac{(\lambda(t-(k-1) T))^{m}}{m !}\right) F_{X_{(k-1) T}^{S} \mid N_{(k-1) T}}\left(M_{n} \mid n\right), \\
& P_{Y, 2}^{R U L}=P\left(N_{(k-1) T} \leq N_{t}<N, X_{(k-1) T}^{S}<M_{N_{(k-1) T}}<L \leq X_{t}^{S}\right)= \\
& \sum_{n=0}^{N-1} \mathrm{e}^{-\lambda(k-1) T} \frac{(\lambda(k-1) T)^{n}}{n !} \int_{0}^{M_{n}} \sum_{m=0}^{N-1-n} \mathrm{e}^{-\lambda(t-(k-1) T)} \frac{(\lambda(t-(k-1) T))^{m}}{m !} \times \\
& \left(1-F_{X_{[(k-1) T, t]}^{S} \mid N_{(k-1) T}, N_{t}-N_{(k-1) T}}(L-x \mid n, m)\right) f_{X_{(k-1) T}^{S} \mid N_{(k-1) T}}(x \mid n) d x,
\end{aligned}
$$

in which $F_{X_{(k-1) T}^{S} \mid N_{(k-1) T}}(\cdot), f_{X_{(k-1) T}^{S} \mid N_{(k-1) T}}(\cdot)$ and $F_{X_{[(k-1) T, t]}^{S} \mid N_{(k-1) T}, N_{t}-N_{(k-1) T}}(\cdot)$ are given by (5), (6) and (7) respectively.

Thus, the long-run expected maintenance cost rates of the $\left(T, \mu_{p}\right)$ strategy and the $\left(T, R_{p}\right)$ strategy are obtained from (19), where the probabilities $P_{p, k}^{R U L}(k T)$ and $P^{R U L}((k-1) T<Y<t)$ are given by (34) and (35) respectively. Optimizing these strategies amounts to determine the couples of decision parameters $\left(T_{o p t}, \mu_{p, o p t}\right)$ and $\left(T_{o p t}, R_{p, o p t}\right)$ which minimize the long-run expected maintenance cost rates

$$
\begin{gathered}
C_{\infty}^{\left(T, \mu_{p}\right)}\left(T_{\text {opt }}, \mu_{p, o p t}\right)=\min _{T>0,0<\mu_{p}<M T T F}\left\{C_{\infty}^{\left(T, \mu_{p}\right)}\left(T, \mu_{p}\right)\right\}, \\
C_{\infty}^{\left(T, R_{p}\right)}\left(T_{\text {opt }}, R_{p, o p t}\right)=\min _{T>0,0<R_{p}<1}\left\{C_{\infty}^{\left(T, R_{p}\right)}\left(T, R_{p}\right)\right\},
\end{gathered}
$$

where MTTF denotes the mean time to failure of the system. Once again, the generalized pattern search algorithm represented in [59] is used to find the optima of the $\left(T, \mu_{p}\right)$ strategy and the $\left(T, R_{p}\right)$ strategy. The cost surface of both these 
strategies as a function of their decision parameters are shown in Fig. 9a and Fig. 9b. The form of the cost rate surfaces justifies the existence of optimal decision parameters. As shown in Fig. 9, for the considered system, the $\left(T, \mu_{p}\right)$ strategy reaches the optimal cost rate $C_{\infty}\left(T_{o p t}, \mu_{p, o p t}\right)=5.7627$ at $T_{o p t}=5$ and $\mu_{p, o p t}=5.5$, and the $\left(T, R_{p}\right)$ strategy reaches the optimal cost rate $C_{\infty}\left(T_{\text {opt }}, R_{p, o p t}\right)=5.7552$ at $T_{\text {opt }}=5$ and $R_{p, o p t}=0.7$.

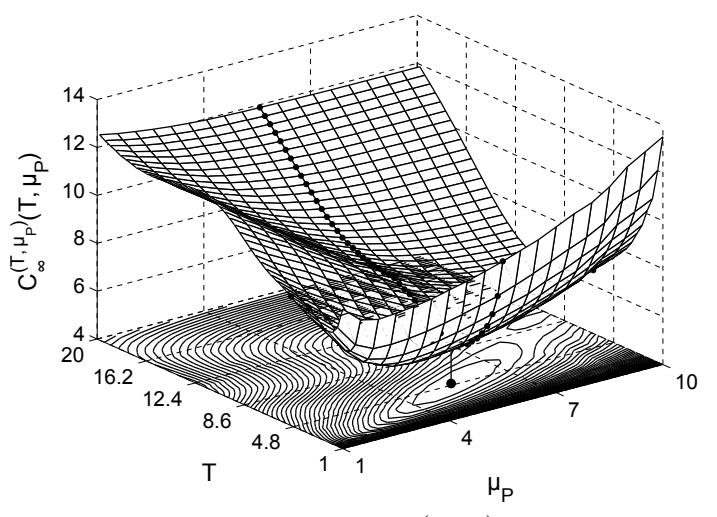

(a) Surface of $C_{\infty}^{\left(T, \mu_{p}\right)}$.

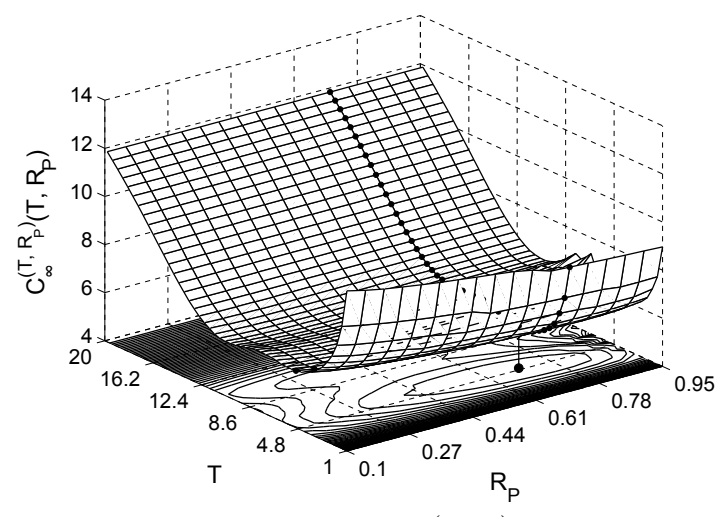

(b) Surface of $C_{\infty}^{\left(T, R_{p}\right)}$.

Fig. 9 - Shape of the cost rates of the $\left(T, \mu_{p}\right)$ strategy and the $\left(T, R_{p}\right)$ strategy.

\section{Performance and Robustness Assessment of Condition-based Maintenance Strategies}

The mathematical cost models developed above help us to quantitatively analyze in this section the performance and robustness of the four proposed CBM strategies. Indeed, through the comparison results on their cost rates in different configurations of maintenance operations costs and system characteristics, we can draw some conclusions on their performance and robustness, and hence find out reliable condition indices for maintenance decision-making.

\subsection{Performance Analysis}

To evaluate the performance of the four considered maintenance strategies, we compare their optimal cost rates in different configuration of maintenance operations costs. The practical constraint $C_{i}<C_{p}<C_{c}$ leads us to fix $C_{c}=100$ and to consider three following case studies

- varied inspection cost: $C_{i}$ varies from 1 to 50 with step equals $1, C_{p}=50$, and $C_{d}=25$,

- varied PR cost: $C_{i}=5, C_{p}$ varies from 5 to 99 with step equals 1 , and $C_{d}=25$,

- varied downtime cost rate: $C_{i}=5, C_{p}=50$, and $C_{d}$ varies from 5 to 100 with step equals 1.

These cost configurations are applied for the system characterized by the set of parameters: $L=50, N=9, \alpha=0.1$, $\beta=0.1$ and $\lambda=0.5$. Fig. 10a, Fig. 10b and Fig. 10c show the results for the three above case studies. In each figure, the lower curve means that the associated strategy has higher performance. We can see clearly that the $(T, M)$ strategy is the least profitable one among the considered strategies, because the sum of crack sizes $X_{t}^{S}$ considered alone cannot completely characterize the system health state. The other strategies allow taking the complete information about the current system health state into maintenance decision-making, so they lead to almost identical cost savings. However, the $\left(T, M, N_{p}\right)$ strategy is not really equivalent to the $\left(T, R_{p}\right)$ strategy and $\left(T, \mu_{p}\right)$ strategy. To clarify this point, we look at the evolution of their optimal decision parameters sketched in Fig. 11a, Fig. 11b and Fig. 11c respectively. In each figure, the subfigures on the top and at the bottom represent respectively the evolutions of the optimal inspection periods $T_{\text {opt }}$ and of the optimal PR thresholds of each strategy (i.e., $M_{o p t}$ and $N_{p, o p t}$ of the $\left(T, M, N_{p}\right)$ strategy, $\mu_{p, o p t}$ of the $\left(T, \mu_{p}\right)$ strategy, and $R_{p, o p t}$ of the $\left(T, R_{p}\right)$ strategy). Note that, for an easier comparison, we have transformed the $M_{o p t}$ and $N_{p, o p t}$ of the $\left(T, M, N_{p}\right)$ strategy, and $\mu_{p, o p t}$ of the $\left(T, \mu_{p}\right)$ strategy into the corresponding conditional system reliabilities using (11) and (12) (i.e., $\left(M_{o p t}, N_{p, o p t}\right) \Rightarrow R\left(t+T_{o p t} \mid M_{o p t}, N_{p, o p t}\right)$ and $\left.\mu_{p, o p t} \Rightarrow\left(x_{\mu_{p, o p t}}, k_{\mu_{p, o p t}}\right) \Rightarrow R\left(t+T_{o p t} \mid x_{\mu_{p, o p t}}, k_{\mu_{p, o p t}}\right)\right)$. One can see from these figures that the values of $T_{\text {opt }}$ of these strategies are almost the same, while $R_{p, o p t}$ is almost equals 


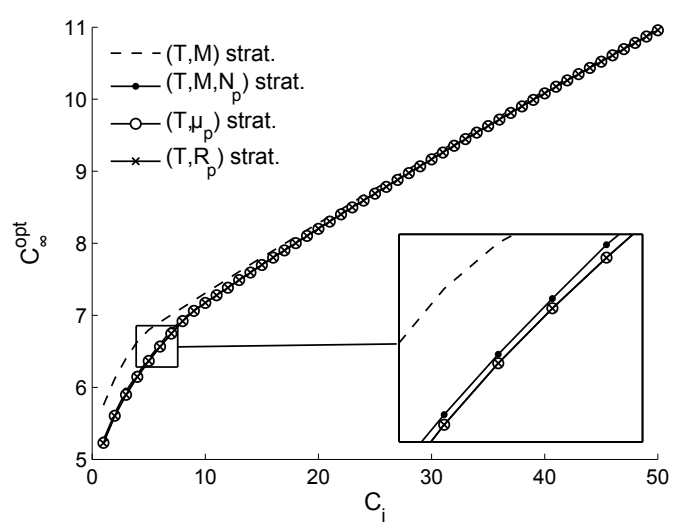

(a) Varied inspection cost.

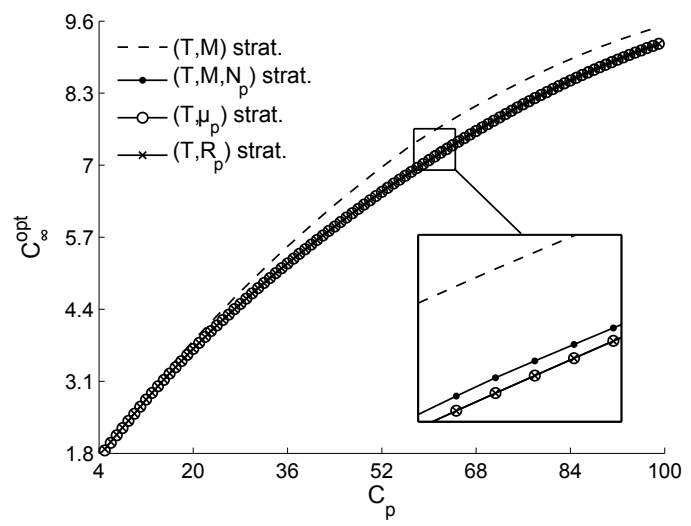

(b) Varied PR cost.

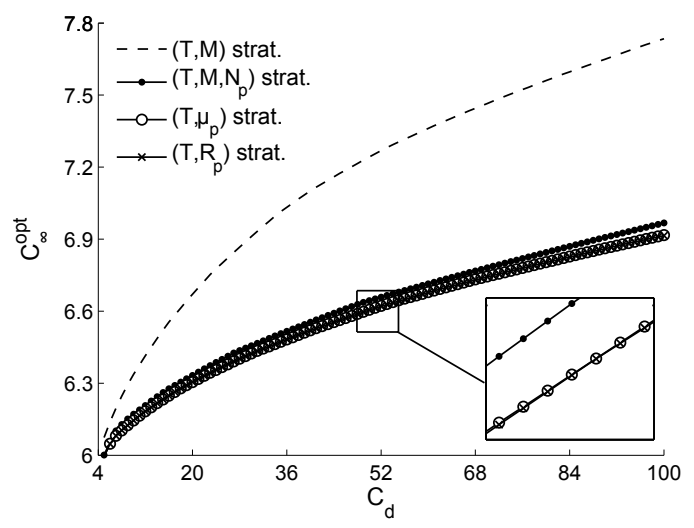

(c) Varied downtime cost rate.

Fig. 10 - Comparison on the performance of the considered maintenance strategies.

to $R\left(t+T_{o p t} \mid x_{\mu_{p, o p t}}, k_{\mu_{p, o p t}}\right)$ and is different than $R\left(t+T_{o p t} \mid M_{o p t}, N_{p, o p t}\right)$. This means that, from the performance viewpoint, the $\left(T, R_{p}\right)$ strategy and the $\left(T, \mu_{p}\right)$ strategy are equivalent, and they differ from the $\left(T, M, N_{p}\right)$ strategy. This remark is also justified by observing the events of PR of the considered strategies in (25) and (33) respectively. More precisely, the optimal long-run expected maintenance cost of the $\left(T, M, N_{p}\right)$ strategy is slightly higher than the $\left(T, R_{p}\right)$ strategy and $\left(T, \mu_{p}\right)$ strategy (see Fig. 10a, Fig. 10b and Fig. 10c). This is because the PR thresholds on the sum of crack size $M$ and on the number of appeared cracks $N_{p}$ are defined independently, and the PR thresholds on the system MRL $\mu_{p}$ and the system conditional reliability $R_{p}$ can contain the information about the dependence between the sum of crack size and the number of cracks. While such a dependence is obviously inherent in the degradation and failure model of the system, it is not surprising that the $\left(T, R_{p}\right)$ strategy and $\left(T, \mu_{p}\right)$ strategy are more profitable than the $\left(T, M, N_{p}\right)$ strategy. Although such a profit is not much significant, in practical applications, we still prefer the $\left(T, R_{p}\right)$ strategy and $\left(T, \mu_{p}\right)$ strategy than the $\left(T, M, N_{p}\right)$ strategy because of their simpler maintenance decision-making structures and parameters optimization. Thus, from the performance viewpoint, it is more interesting to use prognostic condition indices for maintenance decision-making instead of diagnostic condition indices.

\subsection{Robustness Analysis}

The above studies show that the performances in term of the long-run expected average cost rate of the three maintenance strategies $\left(T, M, N_{p}\right),\left(T, \mu_{p}\right)$ and $\left(T, R_{p}\right)$ are almost identical under the assumption of perfect estimation of model parameters. However, in many practical applications, the estimation may be imprecise owing to the lack of data, measurement errors, variability, modeling uncertainties, etc. This leads to errors in determining the optimal decision parameters of a maintenance strategy, and hence a loss in its performance. This section therefore aims at analyzing the impact of such errors on the maintenance cost of these three strategies, and hence giving some conclusions on their robustness. Recall that the robustness refers to the sensitivity of the maintenance cost rate $C_{\infty}$ to the suboptimal choice of decision parameters. As such, looking at the flatness of the optimal zones the $\left(T, \mu_{p}\right)$ strategy and the $\left(T, R_{p}\right)$ strategy (zones around the optimal values of $\mu_{p}$ and $R_{p}$ ) in Fig. 9, it seems that the latter is robuster than the former. However, to objectively assess their robustness, we rely 

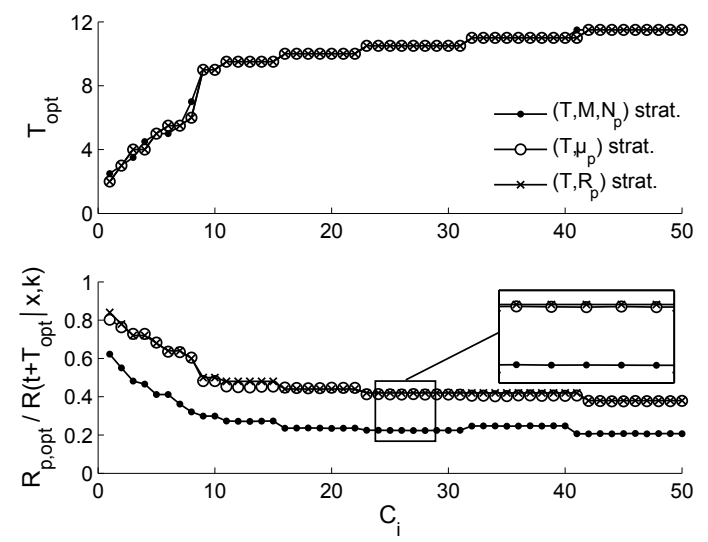

(a) Varied inspection cost.
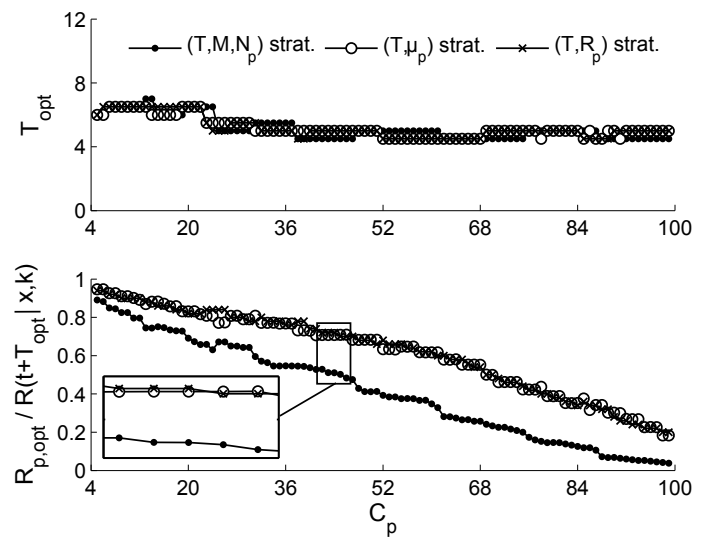

(b) Varied PR cost.
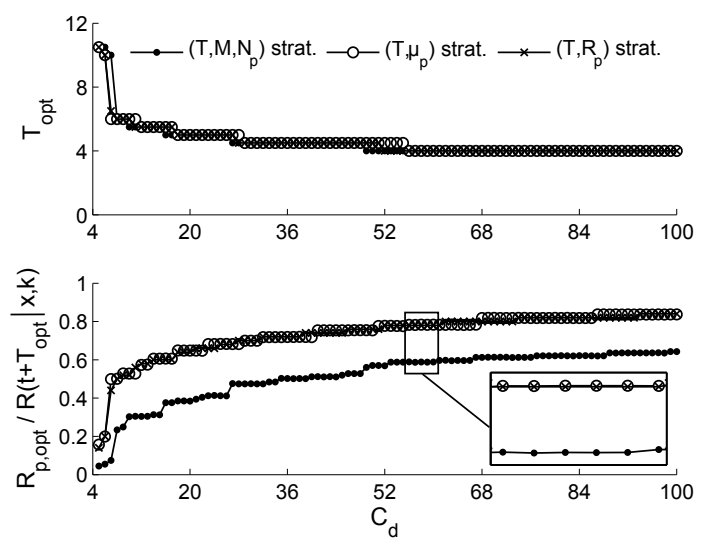

(c) Varied downtime cost rate.

Fig. 11 - Evolution of optimal decision parameters of the considered maintenance strategies.

on a criterion similar to the one proposed in [25]

$$
\epsilon_{C}=\frac{C_{\infty}-C_{\infty}^{o p t}}{C_{\infty}^{o p t}} \times 100 \%
$$

It represents the relative increase in the long-run expected maintenance cost rate of a maintenance strategy when the decision parameters are different than their optimal values. This robustness criterion is then studied with respect to the variability of crack growth behavior.

Specifically, the study is based on the set of maintenance operations costs $C_{i}=5, C_{p}=50, C_{c}=100$ and $C_{d}=25$, and on the system characterized by the set of parameters $L=50, N=9$ and $\lambda=0.5$. Two configurations of crack growth behaviors with the average propagation rate $m=1$ are considered

- small variance rate: $\sigma^{2}=0.1(\alpha=10, \beta=10)$,

- high variance rate: $\sigma^{2}=10(\alpha=0.1, \beta=0.1)$.

Moreover, since the only difference between these three strategies is the way to make the PR decisions, it is sufficient to assess their robustness by analyzing the impact of the suboptimal choice of the PR thresholds on the maintenance cost rate $C_{\infty}$. This is why, for each above configuration, we fix the inspection period $T$ at its optimal value and we vary the other decision parameters (i.e., $M$ for the $\left(T, M, N_{p}\right)$ strategy, $\mu_{p}$ for the $\left(T, \mu_{p}\right)$ strategy, and $R_{p}$ for the $\left(T, R_{p}\right)$ strategy) around their optimal values with a relative error $\epsilon_{p}$ varied from $-20 \%$ to $+20 \%\left(\epsilon_{p}=0\right.$ corresponds to the optimal configurations of the maintenance strategies), and we investigate the evolution of $\epsilon_{C}$. Note that the parameter $N_{p}$ of the $\left(T, M, N_{p}\right)$ strategy, $N_{p} \in \mathbb{N}$, is firstly set at its optimal value. The optimal decision parameters of the considered strategies are shown in Table 5 . Table 6 represents the evolution of the PR thresholds $R_{p}$ and $\mu_{p} \Rightarrow R\left(t+T_{o p t} \mid x_{\mu_{p}}, k_{\mu_{p}}\right)$ with respect to $\epsilon_{p}$. We can remark that with a same $\epsilon_{p}$, the values of $R_{p}$ and $R\left(t+T_{o p t} \mid x_{\mu_{p}}, k_{\mu_{p}}\right)$ are different, and the difference becomes higher for smaller variance of crack growth behavior. This means that the $\left(T, \mu_{p}\right)$ strategy and the $\left(T, R_{p}\right)$ strategy are no longer equivalent when they are out of their optimal configurations, and this is even more true when the variance of crack growth behavior is 
less important.

\begin{tabular}{|c|c|c|}
\hline Config. & Strategy & Optimal decision parameters \\
\hline \hline \multirow{3}{*}{$\sigma^{2}=0.1$} & $\left(T, M, N_{p}\right)$ & $T_{o p t}=5.4, M_{o p t}=22, N_{p, o p t}=5$ \\
\cline { 2 - 3 } & $\left(T, \mu_{p}\right)$ & $T_{o p t}=4.6, \mu_{p, o p t}=5$ \\
\cline { 2 - 3 } & $\left(T, R_{p}\right)$ & $T_{o p t}=4.6, R_{p, o p t}=0.65$ \\
\hline \hline \multirow{3}{*}{$\sigma^{2}=10$} & $\left(T, M, N_{p}\right)$ & $T_{o p t}=4.6, M_{o p t}=23, N_{p}=5$ \\
\cline { 2 - 3 } & $\left(T, \mu_{p}\right)$ & $T_{o p t}=4.6, \mu_{p, o p t}=6$ \\
\cline { 2 - 3 } & $\left(T, R_{p}\right)$ & $T_{o p t}=4.6, R_{p, o p t}=0.7$ \\
\hline
\end{tabular}

Table 5 - Optimal decision parameters of the three considered strategies.

\begin{tabular}{|c|c|ccccccccc|}
\hline Config. & $\epsilon_{p}$ & $-20 \%$ & $-15 \%$ & $-10 \%$ & $-5 \%$ & $0 \%$ & $5 \%$ & $10 \%$ & $15 \%$ & $20 \%$ \\
\hline \hline \multirow{3}{*}{$\sigma^{2}=0.1$} & $\mu_{p}$ & 4 & 4.25 & 4.5 & 4.75 & 5 & 5.25 & 5.5 & 5.75 & 6 \\
\cline { 2 - 11 } & $R\left(t+T_{o p t} \mid x_{\mu_{p}}, k_{\mu_{p}}\right)$ & 0.3552 & 0.3223 & 0.4395 & 0.596 & 0.7009 & 0.7876 & 0.8206 & 0.8946 & 0.9201 \\
\cline { 2 - 10 } & $R_{p}$ & 0.52 & 0.5525 & 0.585 & 0.6175 & 0.65 & 0.6825 & 0.715 & 0.7475 & 0.78 \\
\hline \hline \multirow{3}{*}{$\sigma^{2}=10$} & $\mu_{p}$ & 4.8 & 5.1 & 5.4 & 5.7 & 6 & 6.3 & 6.6 & 6.9 & 7.2 \\
\cline { 2 - 11 } & $R\left(t+T_{o p t} \mid x_{\mu_{p}}, k_{\mu_{p}}\right)$ & 0.4888 & 0.5523 & 0.6009 & 0.6443 & 0.6840 & 0.7008 & 0.7629 & 0.7939 & 0.8145 \\
\cline { 2 - 10 } & $R_{p}$ & 0.56 & 0.595 & 0.63 & 0.665 & 0.7 & 0.735 & 0.77 & 0.805 & 0.84 \\
\hline
\end{tabular}

Table 6 - Evolution of $R_{p}$ and $\mu_{p} \Rightarrow R\left(t+T_{o p t} \mid x_{\mu_{p}}, k_{\mu_{p}}\right)$ with respect to $\epsilon_{p}$.

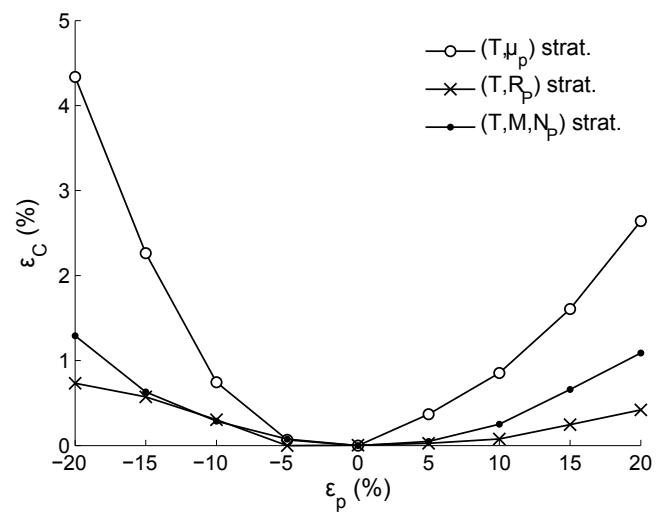

(a) Small variance rate.

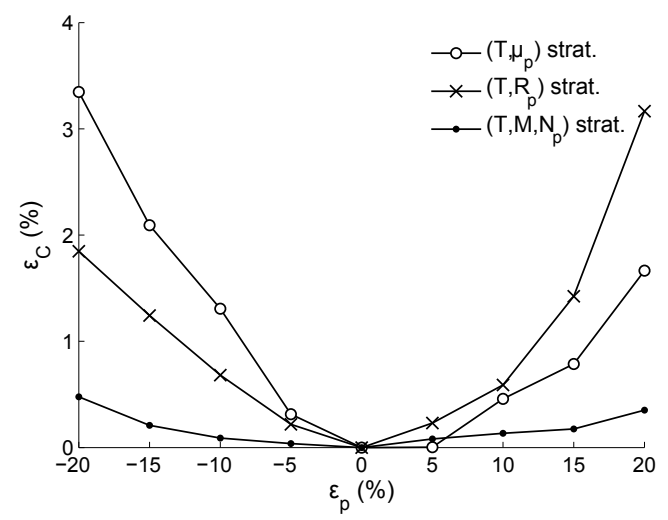

(b) High variance rate.
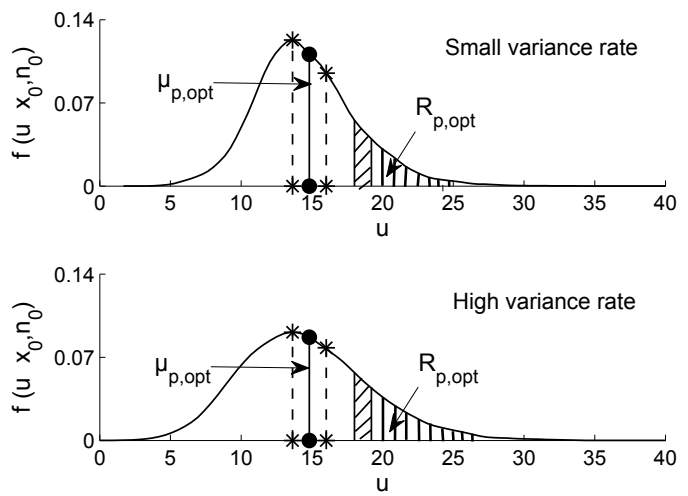

(c) pdf of the conditional RUL of the system.

Fig. 12 - Comparison on the robustness of the considered maintenance strategies.

More precise discussions about the robustness of these two strategies can be drawn from Fig. 12a and Fig. 12b. In these figures, the flatter curve corresponds to robuster strategy. Obviously, the values of $\epsilon_{C}$ increase with respect to the error in decision parameters. Thanks to the nature of the decision parameter (conditional reliability vs. MRL), the $\epsilon_{C}$ curve of the $\left(T, R_{p}\right)$ strategy is generally flatter than the one of the $\left(T, \mu_{P}\right)$ strategy. This can be explained by looking at Figure 12c. In fact, $\mu_{p, o p t}$ corresponds to a point on the curve of the pdf of RUL, and $R_{p, o p t}$ corresponds to area below the curve. With a same error magnitude in a decision parameter, a deviation from a point is intuitively more critical than a deviation from an area. As a result, the $\left(T, R_{p}\right)$ strategy is less sensitive to the parameter error, and hence robuster than the $\left(T, \mu_{p}\right)$ strategy. However, this seems no more true when the variance of crack paths is high (Fig. 12a vs. Fig. 12b). The reason is that the 
curve of the pdf RUL becomes less peaky in this case, and thus the relative deviation of $\mu_{p}$ from its optimal value is less important.

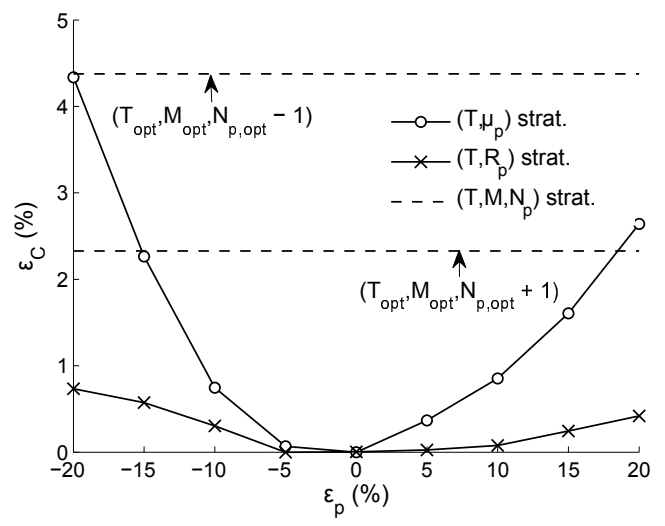

(a) Small variance rate.

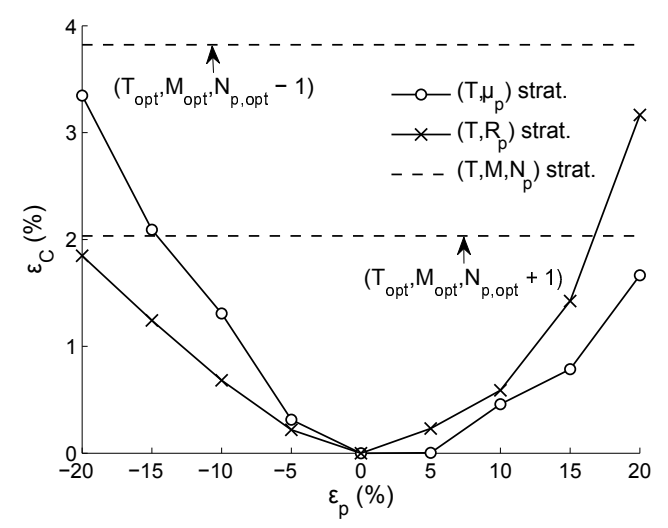

(b) High variance rate.

Fig. 13 - Focusing on the robustness of the $\left(T, M, N_{p}\right)$ strategy.

For the $\left(T, M, N_{p}\right)$ strategy, we can see from Fig. 12a and Fig. 12b that its $\epsilon_{C}$ curves are flat. This means that the $\left(T, M, N_{p}\right)$ strategy remains insensitive to the influence of the variability of $M$. However, it is very sensitive to the change of $N_{p}$. Indeed, observing the $\epsilon_{C}$ curves of the $\left(T, M, N_{p}\right)$ strategy when $N_{p}=N_{p, o p t} \pm 1, T=T_{o p t}$ and $M=M_{o p t}$ in Figure 13 , even with one unit of deviation in $N_{p}$ from its optimum, the value of $\epsilon_{C}$ increases significantly and become much higher than the ones of the $\left(T, \mu_{p}\right)$ strategy and the $\left(T, R_{p}\right)$ strategy. As such, in general, the $\left(T, M, N_{p}\right)$ strategy is less robust than the $\left(T, \mu_{p}\right)$ strategy and the $\left(T, R_{p}\right)$ strategy. In other words, using the prognostic condition indices instead of diagnostic ones can lead to robuster maintenance decisions.

\section{Conclusions and Perspectives}

The present paper considers a system subject to competing risks due to multiple degradation paths of SCC which appear on different passive components or structures. A degradation and failure model based on a combination of Poisson process and Gamma processes is developed to describe the system behavior. Compared to the existing models, the way to define the system failure in our model makes it more realistic and reliable for safety and reliability analysis. Furthermore, it can be easily extended to be a new element in the class of DTS reliability modes. Based on this competing risks model, two prognostic condition indices (i.e., the average-based RUL and the distribution-based RUL indices) are synthesized, and the associated maintenance cost models are also developed. These models are optimized and compared with the ones whose maintenance decision are based on diagnostic condition indices. The differences among the considered CBM strategies lie in the way the knowledge on the degradation model is used, either implicitly in the optimization process when the diagnostic condition indices are used, or more explicitly with the diagnostic prognostic indices. The CBM strategy implemented without using the full information on the degradation model, would be for sure not optimal, and would incur a greater maintenance cost. However, it does not make a significant difference to use this information either implicitly or explicitly as far as the expected maintenance cost is considered, and not surprisingly all the strategies using correctly the same amount of information lead more or less to the same expected maintenance cost. But this is true only under the assumption of a perfect knowledge of the degradation model. The work shows that using explicitly the knowledge of the degradation model by building prognostic condition indices (instead of using it only implicitly in the maintenance optimization) leads to robuster maintenance strategies. This robustness property is very important for a practical implementation of the strategy since most often the degradation phenomenon is not perfectly modeled. Furthermore, between the two considered prognostic indices, the distribution-based RUL index is more informative and reliable for maintenance decision-making than the average-based RUL index, because it can lead to robuster maintenance strategy. This result therefore encourages us to invest more strongly in prognosis methods which can give a probability law of future system heath state. The work in this paper also convinces us that the value of prognostic information can be only properly assessed in light of the downstream maintenance decision process and of the overall maintenance performance and robustness. 
For our future works, we continue studying the effectiveness of diagnostic and prognostic condition indices based on other degradation and failure models of SCC. For instance, we can consider a model whose the system failure occurs when there are at least some number of cracks bigger than some length.

\section{Appendix A. Proof of (5)}

$X_{t}^{S} \mid N_{t}$ is a random variable representing the sum of all crack increments in the time intervals $[0, t]$ given the number of cracks up to time $t, N_{t}$, its cdf can be computed as

$$
\begin{aligned}
F_{X_{t}^{S} \mid N_{t}}(x \mid k)=P & \left(X_{t}^{S}<x \mid N_{t}=k\right)= \\
& \int_{0}^{t} \int_{t_{1}}^{t} \cdots \int_{t_{k-1}}^{t} P\left(X_{t}^{S}<x \mid T_{1}=t_{1}, \cdots, T_{k}=t_{k}, N_{t}=k\right) f_{T_{1}, \cdots, T_{k} \mid N_{t}}\left(t_{1}, \cdots, t_{k} \mid k\right) d t_{1} d t_{2} \cdots d t_{n} .
\end{aligned}
$$

From $(2), F_{X_{t}^{S} \mid N_{t}}(x \mid k)$ is rewritten as

$$
\begin{aligned}
& F_{X_{t}^{S} \mid N_{t}}(x \mid k)=\int_{0}^{t} \int_{t_{1}}^{t} \cdots \int_{t_{n-1}}^{t} F_{\alpha\left(n t-\sum_{i=1}^{n} t_{i}\right), \beta}(x) \frac{n !}{t^{n}} d t_{1} d t_{2} \cdots d t_{n}= \\
& \int_{0}^{t} \int_{0}^{t} \cdots \int_{0}^{t} F_{\alpha\left(n t-\sum_{i=1}^{n} t_{i}\right), \beta}(x) \frac{1}{t^{n}} d t_{1} d t_{2} \cdots d t_{n}=E_{\Theta}\left[F_{\alpha\left(k t-\sum_{i=1}^{k} \theta_{i}\right), \beta}(x)\right],
\end{aligned}
$$

where $F_{\alpha \cdot\left(k t-\sum_{i=1}^{k} t_{i}\right), \beta}(x)$ is derived from (4), $E_{\Theta}[\cdot]$ denotes the expectation with respect to the random vector $\Theta=$ $\left\{\theta_{1}, \ldots, \theta_{k}\right\}$ whose each element follows a uniform distribution $\theta_{j} \sim U(0, t), j=1, \ldots, k$.

\section{Appendix B. Proof of (7)}

$X_{[t, t+u]}^{S} \mid N_{t}, N_{t+u}-N_{t}$ is a random variable representing the sum of crack increments in the time intervals $[t, t+u]$ given the number of cracks appeared at time $t, N_{t}$, and the additional number of cracks in the time interval $[t, t+u], N_{t+u}-N_{t}$. Knowing $N_{t}=k$ and $N_{t+u}-N_{t}=h$, this variable can be decomposed by to parts

- the increase of the $k$ old cracks appeared before $t$. This quantity follows a Gamma probability law with parameters $\alpha k t$ and $\beta$,

- the increase of the $h$ new cracks appeared in the time interval $[t, t+u]$. This quantity follows a Gamma probability law with parameters $\alpha\left(h(t+u)-\sum_{i=k+1}^{k+h} T_{i}\right)$ and $\beta$, where $T_{k+1}, T_{k+2}, \ldots, T_{k+h}$ are the arrival times of cracks in the time interval $[t, t+u]$.

As such, if $t_{i}, i=k+1, \ldots, k+h$, is a realization of the random variable $T_{i}\left(t<t_{k+1}<t_{k+2}<\cdots<t_{k+h}<t+u\right)$, then the cdf of $X_{[t, t+u]}^{S} \mid N_{t}, N_{t+u}-N_{t}$ can be computed as

$$
\begin{aligned}
& F_{X_{[t, t+u]}^{S} \mid N_{t}, N_{t+u}-N_{t}}(x \mid k, h)=P\left(X_{[t, t+u]}^{S}<x \mid N_{t}=k, N_{t+u}-N_{t}=h\right)= \\
& \int_{t}^{t+u} \int_{t_{k+1}}^{t+u} \cdots \int_{t_{k+h-1}}^{t+u} P\left(X_{[t, t+u]}^{S}<x \mid N_{t}=k, T_{k+1}=t_{k+1}, \cdots, T_{k+h}=t_{k+h}\right) \times \\
& \quad f_{T_{k+1}, \ldots, T_{k+h} \mid N_{t+u}-N_{t}}\left(t_{k+1}, t_{k+2}, \cdots, t_{k+h} \mid h\right) d t_{k+1} d t_{k+2} \cdots d t_{k+h} .
\end{aligned}
$$

From $(2), F_{X_{[t, t+u]}^{S} \mid N_{t}, N_{t+u}-N_{t}}(x \mid k, h)$ is rewritten as

$$
F_{X_{[t, t+u]}^{S} \mid N_{t}, N_{t+u}-N_{t}}(x \mid k, h)=\int_{t}^{t+u} \int_{t_{k+1}}^{t+u} \cdots \int_{t_{k+h-1}}^{t+u} F_{\alpha\left(k t+h(t+u)-\sum_{i=k+1}^{k+h} t_{i}\right), \beta}(x) \frac{h !}{t^{h}} d t_{k+1} d t_{k+2} \cdots d t_{k+h}
$$


Let's denote $l_{i}=t_{k+i}-t$,

$$
\begin{aligned}
F_{X_{[t, t+u]}^{S} \mid N_{t}, N_{t+u}-N_{t}}(x \mid k, h) & =\int_{u}^{t} \int_{l_{1}}^{t} \cdots \int_{l_{h-1}}^{t} F_{\alpha\left((k+h) t-\sum_{i=1}^{h} l_{i}\right), \beta}(x) \frac{h !}{t^{h}} d l_{1} d l_{2} \cdots d l_{h}= \\
& \int_{0}^{t} \int_{0}^{t} \cdots \int_{0}^{t} F_{\alpha\left((k+h) t-\sum_{i=1}^{h} l_{i}\right), \beta}(x) \frac{1}{t^{h}} d l_{1} d l_{2} \cdots d l_{h}=E_{\Theta}\left[F_{\alpha\left((k+h) u-\sum_{i=1}^{h} \theta_{i}\right), \beta}(x)\right]
\end{aligned}
$$

where $F_{\alpha\left((k+h) u-\sum_{i=1}^{h} \theta_{i}\right), \beta}(x)$ is derived from $(4), E_{\Theta}[\cdot]$ denotes the expectation with respect to the random vector $\Theta=$ $\left\{\theta_{1}, \ldots, \theta_{h}\right\}$ whose each element follows a uniform distribution $\theta_{j} \sim U(0, t), j=1, \ldots, h$.

\section{Appendix C. Proof of the monotonicity of the system conditional reliability $R(t+u \mid x, k)$}

- For a fixed $k, 0 \leq k \leq N$, the conditional reliability $R(t+u \mid x, k)$ is non-increasing in $x$.

We have to prove that, given a fixed $k, 0 \leq k \leq N$, and $x_{1} \leq x_{2}$, then $R\left(t+u \mid x_{1}, k\right) \geq R\left(t+u \mid x_{2}, k\right)$. Indeed, when $x_{1} \leq x_{2}, L-x_{1} \geq L-x_{2}$, and thus $F_{X_{[t, t+u]}^{S} \mid N_{t}, N_{t+u}-N_{t}}\left(L-x_{1} \mid k, h\right) \geq F_{X_{[t, t+u]}^{S} \mid N_{t}, N_{t+u}-N_{t}}\left(L-x_{2} \mid k, h\right)$. Consequently, one can derive from (11) that $R\left(t+u \mid x_{1}, k\right) \geq R\left(t+u \mid x_{2}, k\right)$.

- For a fixed $x, 0 \leq x \leq L$, the conditional reliability $R(t+u \mid x, k)$ is non-increasing in $k$.

We have to prove that, given a fixed $x, 0 \leq x \leq L$, and $k_{1} \leq k_{2}$, then $R\left(t+u \mid x, k_{1}\right) \geq R\left(t+u \mid x, k_{2}\right)$. Indeed, we have from (7) that $F_{X_{[t, t+u]}^{S} \mid N_{t}, N_{t+u}-N_{t}}(L-x \mid k, h)=E_{\Theta}\left[F_{\alpha\left((k+h) u-\sum_{i=1}^{h} \theta_{i}\right), \beta}(L-x)\right]$ where $\Theta=\left\{\theta_{1}, \ldots, \theta_{h}\right\}$ and $\theta_{j} \sim U(0, t), j=1, \ldots, h$. Since $k_{1} \leq k_{2}, F_{\alpha\left(\left(k_{1}+h\right) u-\sum_{i=1}^{h} \theta_{i}\right), \beta}(L-x) \geq F_{\alpha\left(\left(k_{2}+h\right) u-\sum_{i=1}^{h} \theta_{i}\right), \beta}(L-x)$, and thus $F_{X_{[t, t+u]}^{S} \mid N_{t}, N_{t+u}-N_{t}}\left(L-x \mid k_{1}, h\right) \geq F_{X_{[t, t+u]}^{S} \mid N_{t}, N_{t+u}-N_{t}}\left(L-x \mid k_{2}, h\right)$. Consequently, according to $(11), R\left(t+u \mid x, k_{1}\right) \geq$ $R\left(t+u \mid x, k_{2}\right)$.

\section{Acknowledgements}

This work has been partially supported by the LabEx PERSYVAL-Lab (ANR-11-LABX-0025-01) funded by the French program Investissement d'avenir.

[1] R. Ahmad and S. Kamaruddin, "An overview of time-based and condition-based maintenance in industrial application," Computers 83 Industrial Engineering, vol. 63, no. 1, pp. 135-149, 2012.

[2] K. T. Huynh, S. T. Mai, A. Grall, and C. Bérenguer, "Towards a reliable condition index for condition-based maintenance decisionmaking," in Proc. of 2nd International Conference on Control and Fault-Tolerant Systems - SysTol 2013, 2013, pp. 826-831, doi:10.1109/SysTol.2013.6693923.

[3] H. C. Pusey and M. J. Roemer, "An assessment of turbomachinery condition monitoring and failure prognosis technology," Shock and Vibration Digest, vol. 31, pp. 365-371, 1999.

[4] A. K. S. Jardine, D. Lin, and D. Banjevic, "A review on machinery diagnostics and prognostics implementing condition-based maintenance," Mechanical systems and signal processing, vol. 20, no. 7, pp. 1483-1510, 2006.

[5] J. Lee, F. Wu, W. Zhao, M. Ghaffari, L. Liao, and D. Siegel, "Prognostics and health management design for rotary machinery systems reviews, methodology and applications," Mechanical Systems and Signal Processing, vol. 42, no. 1, pp. 314-334, 2014.

[6] R. Ahmad and S. Kamaruddin, "A review of condition-based maintenance decision-making," European Journal of Industrial Engineering, vol. 6, no. 5, pp. 519-541, 2012.

[7] M. Shafiee, S. Duffuaa, and S. Duffuaa, "Maintenance strategy selection problem: an mcdm overview," Journal of Quality in Maintenance Engineering, vol. 21, no. 4, 2015.

[8] Y. Peng, M. Dong, and M. J. Zuo, "Current status of machine prognostics in condition-based maintenance: a review," The International Journal of Advanced Manufacturing Technology, vol. 50, no. 1-4, pp. 297-313, 2010.

[9] X. S. Si, W. Wang, C. H. Hu, and D. H. Zhou, "Remaining useful life estimation - a review on the statistical data driven approaches," European Journal of Operational Research, vol. 213, no. 1, pp. 1-14, 2011.

[10] J. Z. Sikorskaa, M. Hodkiewicz, and L. Ma, "Prognostic modelling options for remaining useful life estimation by industry," Mechanical Systems and Signal Processing, vol. 25, no. 5, pp. 1803-1836, 2011.

[11] W. Wang, "Overview of a semi-stochastic filtering approach for residual life estimation with applications in condition based maintenance," Proceedings of the Institution of Mechanical Engineers, Part O: Journal of Risk and Reliability, vol. 225, no. 2, pp. 185-197, 2011.

[12] J. Zaytoon and S. Lafortune, "Overview of fault diagnosis methods for discrete event systems," Annual Reviews in Control, vol. 37, no. 2, pp. 308-320, 2013.

[13] L. Travé-Massuyès, "Bridging control and artificial intelligence theories for diagnosis: A survey," Engineering Applications of Artificial Intelligence, vol. 27, pp. 1-16, 2014. 
[14] L. Liao and F. Kottig, "Review of hybrid prognostics approaches for remaining useful life prediction of engineered systems, and an application to battery life prediction," IEEE Transactions on Reliability, vol. 63, no. 1, pp. 191-207, 2014.

[15] J. H. Shin and H. B. Jun, "On condition based maintenance policy," Journal of Computational Design and Engineering, vol. 2, no. 2, pp. $119-127,2015$.

[16] H. M. Wagner, Principles of Operations Research. Prentice-Hall, Englewood Cliffs, N.J., 1975.

[17] C. Bérenguer, "On the mathematical condition-based maintenance modelling for continuously deteriorating systems," International Journal of Materials and Structural Reliability, vol. 6, no. 2, pp. 133-151, 2008.

[18] K. T. Huynh, A. Barros, and C. Bérenguer, "Multi-level decision-making for the predictive maintenance of k-out-of-n:f deteriorating systems," IEEE Transactions on Reliability, vol. 64, no. 1, pp. 94-117, 2015.

[19] J. M. van Noortwijk, "A survey of the application of gamma processes in maintenance," Reliability Engineering \& System Safety, vol. 94, no. 1 , pp. 2-21, 2009 .

[20] E. Deloux, B. Castanier, and C. Bérenguer, "Predictive maintenance policy for a gradually deteriorating system subject to stress," Reliability Engineering 85 System Safety, vol. 94, no. 2, pp. 418-431, 2009

[21] K. T. Huynh, A. Barros, and C. Bérenguer, "Adaptive condition-based maintenance decision framework for deteriorating systems operating under variable environment and uncertain condition monitoring," Proceedings of the Institution of Mechanical Engineers, Part O: Journal of Risk and Reliability, vol. 226, no. 6, pp. 602-623, 2012.

[22] K. T. Huynh, A. Barros, and C. Bérenguer, "A reliability-based opportunistic predictive maintenance model for k-out-of-n deteriorating systems," Chemical Engineering Transactions, vol. 33.

[23] K. T. Huynh, I. T. Castro, A. Barros, and C. Bérenguer, "On the use of mean residual life as a condition index for condition-based maintenance decision-making," IEEE Transactions on Systems, Man, and Cybernetics: Systems, vol. 44, no. 7, pp. 877-893, 2014.

[24] N. C. Caballé, I. T. Castro, C. J. Pérez, and J. M. Lanza-Gutiérrez, "A condition-based maintenance of a dependent degradation-thresholdshock model in a system with multiple degradation processes," Reliability Engineering E System Safety, vol. 134, pp. 98-109, 2015.

[25] L. M. Maillart and S. M. Pollock, "The effect of failure-distribution specification-errors on maintenance costs," in Proc. of Annual Reliability and Maintainability Symposium - RAMS 1999, 1999, pp. 69-77, doi:10.1109/RAMS.1999.744099.

[26] C. Blain, A. Barros, A. Grall, and Y. Lefebvre, "Modelling of stress corrosion cracking with stochastic processes-application to steam generators," in Pro. of the European Safety and Reliability Conference - ESREL 2007, 2007, pp. 2395-2400.

[27] I. T. Castro, A. Barros, and A. Grall, "Age-based preventive maintenance for passive components submitted to stress corrosion cracking," Mathematical and Computer Modelling, vol. 54, no. 1, pp. 598-609, 2011.

[28] H. C. Tijms, A first course in stochastic models. Wiley, New York, 2003.

[29] J. M. Boursier, D. Desjardins, and F. Vaillant, "The influence of the strain-rate on the stress corrosion cracking of alloy 600 in high temperature primary water," Corrosion science, vol. 37, no. 3, pp. 493-508, 1995.

[30] A. Grall, L. Dieulle, C. Bérenguer, and M. Roussignol, "Continuous-time predictive-maintenance scheduling for a deteriorating system," IEEE Transactions on Reliability, vol. 51, no. 2, pp. 141-150, 2002.

[31] K. T. Huynh, A. Barros, and C. Bérenguer, "Maintenance decision-making for systems operating under indirect condition monitoring: Value of online information and impact of measurement uncertainty," IEEE Transactions on Reliability, vol. 61, no. 2, pp. 410-425, 2012.

[32] I. T. Castro, "An age-based maintenance strategy for a degradation-threshold-shock-model for a system subjected to multiple defects," Asia-Pacific Journal of Operational Research, vol. 30, no. 5, 2013.

[33] I. T. Castro, N. C. Caballé, and C. J. Pérez, "A condition-based maintenance for a system subject to multiple degradation processes and external shocks," International Journal of Systems Science, 2013, doi:10.1080/00207721.2013.828796.

[34] A. Valor, F. Caleyo, L. Alfonso, D. Rivas, and J. M. Hallen, "Stochastic modeling of pitting corrosion: a new model for initiation and growth of multiple corrosion pits," Corrosion Science, vol. 49, no. 2, pp. 559-579, 2007.

[35] B. Castanier and T. G. Yeung, "Optimal highway maintenance policies under uncertainty," in Proc. of Annual Reliability and Maintainability Symposium - RAMS 2008, 2008, pp. 25-30, doi:10.1109/RAMS.2008.4925764.

[36] S. Mercier, L. Bordes, E. Remy, and E. Dautrême, "A stochastic model for competing degradations," Pro. of the European Safety and Reliability Conference - ESREL 2013, pp. 1731-1738, 2013.

[37] N. D. Singpurwalla, "Survival in dynamic environments," Statistical Science, vol. 10, no. 1, pp. 86-103, 1995.

[38] C. Cocozza-Thivent, Processus stochastiques et fiabilité des systèmes. Springer, 1997.

[39] K. T. Huynh, A. Barros, C. Bérenguer, and I. T. Castro, "A periodic inspection and replacement policy for systems subject to competing failure modes due to degradation and traumatic events," Reliability Engineering ES System Safety, vol. 96, no. 4, pp. 497-508, 2011.

[40] A. Bobet, "Modelling of crack initiation, propagation andcoalescence in uniaxial compression," Rock Mechanics and Rock Engineering, vol. 33, no. 2, pp. 119-139, 2000.

[41] M. Kamaya and T. Haruna, "Crack initiation model for sensitized 304 stainless steel in high temperature water," Corrosion science, vol. 48, no. 9, pp. 2442-2456, 2006 .

[42] A. Lehmann, "Joint modeling of degradation and failure time data," Journal of Statistical Planning and Inference, vol. 139, no. 5, pp. 1693-1706, 2009.

[43] G. A. Klutke and Y. Yang, "The availability of inspected systems subject to shocks and graceful degradation," IEEE Transactions on Reliability, vol. 51, no. 3, pp. 371-374, 2002.

[44] W. Li and H. Pham, "Reliability modeling of multi-state degraded systems with multi-competing failures and random shocks," IEEE Transactions on Reliability, vol. 54, no. 2, pp. 297-303, 2005. 
[45] Y. Wang and H. Pham, "Dependent competing-risk degradation systems," in Safety and Risk Modeling and Its Applications, ser. Springer Series in Reliability Engineering. Springer, 2011, pp. 197-218.

[46] Y. Wang and H. Pham, "A multi-objective optimization of imperfect preventive maintenance policy for dependent competing risk systems with hidden failure," IEEE Transactions on Reliability, vol. 60, no. 4, pp. 770-781, 2011.

[47] H. Peng, Q. Feng, and D. W. Coit, "Reliability and maintenance modeling for systems subject to multiple dependent competing failure processes," IIE Transactions, vol. 43, no. 1, pp. 12-22, 2011.

[48] L. Jiang, Q. Feng, and D. W. Coit, "Reliability and maintenance modeling for dependent competing failure processes with shifting failure thresholds," IEEE Transactions on Reliability, vol. 61, no. 4, pp. 932-948, 2012.

[49] K. Rafiee, Q. Feng, and D. W. Coit, "Reliability modeling for dependent competing failure processes with changing degradation rate," IIE Transactions, vol. 46, no. 5, pp. 483-496, 2014.

[50] K. T. Huynh, I. T. Castro, A. Barros, and C. Bérenguer, "Modeling age-based maintenance strategies with minimal repairs for systems subject to competing failure modes due to degradation and shocks," European Journal of Operational Research, vol. 218, no. 1, pp. 140-151, 2012.

[51] X. Liu, J. Li, K. Al-Khalifa, A. S. Hamouda, D. W. Coit, and E. A. Elsayed, "Condition-based maintenance for continuously monitored degrading systems with multiple failure modes," IIE Transactions, vol. 45, no. 4, pp. 422-435, 2013.

[52] A. Heng, S. Zhang, A. C. C. Tan, and J. Mathew, "Rotating machinery prognostics: State of the art, challenges and opportunities," Mechanical Systems and Signal Processing, vol. 23, no. 3, pp. 724-739, 2009.

[53] K. Le Son, M. Fouladirad, A. Barros, E. Levrat, and B. Iung, "Remaining useful life estimation based on stochastic deterioration models: A comparative study," Reliability Engineering \& System Safety, vol. 112, pp. 165-175, 2013.

[54] H. Wang, "A survey of maintenance policies of deteriorating systems," European Journal of Operational Research, vol. 139, no. 3, pp. 469-489, 2002.

[55] C. T. Lam and R. H. Yeh, "Optimal maintenance-policies for deteriorating systems under various maintenance strategies," IEEE Transactions on Reliability, vol. 43, no. 3, pp. 423-430, 1994.

[56] H. Kawai, J. Koyanagi, and M. Ohnishi, "Optimal maintenance problems for markovian deteriorating systems," in Stochastic Models in Reliability and Maintenance. Springer, 2002, pp. 193-218.

[57] M. Abdel-Hameed, Lévy Processes and Their Applications in Reliability and Storage. Springer, 2014.

[58] B. J. Lee, C. W. Kang, S. J. Kim, and S. J. Bae, "Optimal replacement strategy for stochastic deteriorating system with random wear limit under periodic inspections," The International Journal of Advanced Manufacturing Technology, vol. 71, no. 1-4, pp. 219-231, 2014.

[59] C. Audet, J. Dennis, and E. John, "Analysis of generalized pattern searches," SIAM Journal on Optimization, vol. 13, no. 3, pp. 889-903, 2002. 